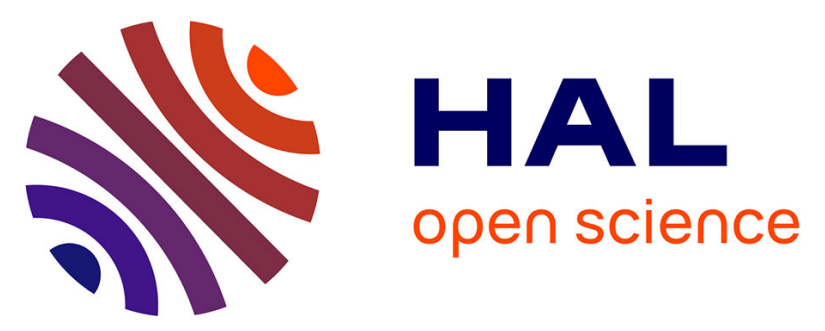

\title{
Ionic parameters identification of an inverse problem of strongly coupled PDE's system in cardiac electrophysiology using Carleman estimates
}

Yassine Abidi, Mourad Bellassoued, Moncef Mahjoub, Nejib Zemzemi

\section{- To cite this version:}

Yassine Abidi, Mourad Bellassoued, Moncef Mahjoub, Nejib Zemzemi. Ionic parameters identification of an inverse problem of strongly coupled PDE's system in cardiac electrophysiology using Carleman estimates. Mathematical Modelling of Natural Phenomena, 2019, Mathematical Modelling in Cardiology, 14 (2), 10.1051/mmnp/2018060 . hal-01923862

\section{HAL Id: hal-01923862 \\ https://hal.inria.fr/hal-01923862}

Submitted on 15 Nov 2018

HAL is a multi-disciplinary open access archive for the deposit and dissemination of scientific research documents, whether they are published or not. The documents may come from teaching and research institutions in France or abroad, or from public or private research centers.
L'archive ouverte pluridisciplinaire HAL, est destinée au dépôt et à la diffusion de documents scientifiques de niveau recherche, publiés ou non, émanant des établissements d'enseignement et de recherche français ou étrangers, des laboratoires publics ou privés. 


\title{
IONIC PARAMETERS IDENTIFICATION OF AN INVERSE PROBLEM OF STRONGLY COUPLED PDE'S SYSTEM IN CARDIAC ELECTROPHYSIOLOGY USING CARLEMAN ESTIMATES
}

\author{
Yassine Abidi $^{1}$, Mourad Bellassoued $^{1}$, Moncef $_{\text {Mahjoub }}{ }^{1}$ and Nejib Zemzemi ${ }^{2,3}$
}

\begin{abstract}
In this paper, we consider an inverse problem of determining multiple ionic parameters of a $2 \times 2$ strongly coupled parabolic-elliptic reaction-diffusion system arising in cardiac electrophysiology modelling. We use the bidomain model coupled to an ODE system and we consider a general formalism of physiologicaly-detailed cellular membrane models to describe the ionic exchanges at the microscopic level. Our main result is the uniqueness and a Lipschitz stability estimate of the ion channels conductance parameters of the model using subboundary observations over an interval of time. The key ingredients are a global Carleman-type estimate with a suitable observations acting on a part of the boundary.
\end{abstract}

Mathematics Subject Classification. 35Q92,34A55.

November 15,2018

\section{INTRODUCTION}

The electric wave in the heart is governed by a system of reaction-diffusion partial differential equations called the bidomain model. This system is coupled nonlinearly to an ordinary differential equations (ODEs) modeling the cellular membrane dynamics. The cellular membrane electrical activity model is based on the Hodgkin and Huxley (HH) formalism [25] which has been adapted to cardiac Purkinje cells by Denis Nobel [39]. After that, many other model have been introduced to describe the electrical activity of the cell membrane in the myocardium. In 1977, Beeler and Reuter [5] introduced a ventricular cells model. Di Francesco and Noble proposed in 1985 a model that takes into account ion pumps, which allows different chemical species such as the sodium potassium and calcium to regain their stable states [22]. Rudy and his collaborators proposed series of models based on the $\mathrm{HH}$ formalism which are chronologically more and more complex $[34,36,37,40,45]$ and take into account more and more the physiological behaviors of the ion channels. Other models like [12,16,46] have been also extensively used in the computational electrophysiology community. In all these models, the ion channels maximal conductance parameter plays an important role in generating the action potential but

Keywords. Lipschitz stability estimate, carleman estimate, cardiac electrophysiology, bidomain system, physiological ionic model, ionic parameters.

1 Tunis El Manar University, ENIT-LAMSIN, BP 37, Le Belvédère 1002 Tunis, Tunisia. e-mail: yassine.abidi@enit.rnu.tn

\& mourad.bellassoued@enit.utm.tn \& moncef.mahjoub@enit.rnu.tn.

2 INRIA, Bordeaux Sud-Ouest, 200 Avenue de la vielle Tour 33405 Talence Cedex France. e-mail: nejib.zemzemi@inria.fr.

3 IHU LIRYC, Electrophysiology and Heart Modeling Institute, Pessac, France. 
also to consider some pathological conditions or when taking into account the effect of drugs. In [38], authors studied the sensitivity of the strength and duration of the action potential in the parameters describing the sodium channel. In [54], authors demonstrate the ability of computational models to simulate the effect of drug action on the electrical activity of the heart, at the level of the ion-channel, cell, heart and ECG body surface potential. In [41,42], authors showed how, with few changes in the maximal conductances of the ion currents, they reproduced different control action potentials. In [1], the authors propose a mathematical approach for the analysis of drugs effects on the electrical activity of hiPSC-CMs based on multi-electrode array experiments where the drug acts directly on the maximal conductance of the targeted ion channel. All these examples demonstrate the importance of identifying or estimating the maximal conductance parameters when dealing with numerical simulations in multi-scale models. Despite their importance, ion channel maximal conductances have not been subject of abondant theoretical studies. The only theoretical work that we know is [2], which proves under certain conditions the stability of the ionic conductance parameters identification problem in case of the monodomain approximation. Two other works has been dedicated to the parameters of phenomenological ionic models still in the case of the monodomain approximation. In [10], authors showed Lipschitz stability inequalities for the identification of some parameters of the FitzHugh-Nagumo model from measurements on the cardiac potential and the ionic variable. The paper [33] shows the stability of the identification a reaction parameter in the Mitchell-Schaeffer model for the monodomain system. To the best of our knowledge no work proves the stability of the ion channels conductances in the case of the bidomain model. This would be the subject of our paper.

In the framework of the bidomain model, there some works that have been dedicated to study the stability of the cardiac tissue conductivities identifiability problem. The paper by Ainseba et al. [4] obtains the stability results for the conductivities diffusion coefficients to a strongly reaction-diffusion system modeling electrical activity in the heart using Carleman estimate. But their method is based on an approximation of the monodomain model and establish a Carleman estimate for a family of parabolic equations. Recently, Wu and Yu [52] convert the strongly coupled terms to the derivatives of the state variables components. The authors establish a Hölder stability result for the inverse conductivities problem for a linearized reaction term in the bidomain model in electrophysiology where the conductivities are scalar functions. However, considering a linear reaction term significantly reduces the complexity of the problem. Authors also consider a linear and simple approximation of the dynamic system governing the cell membrane activity at the microscopic level. Both simplification are non-realistic but also non phenomenologically based models in the sense that one could not generate the shape of an action potential with these models. However, the trick that they proposed to weaken the coupling in the bidomain system is very interesting and gives a good way to deal with a strongly coupled problem.

Strongly coupled systems are notoriously difficult to treat. Many of the standard results are not valid for a strongly coupled system. For example, there is no maximum principle nor DeGiorgi-Nash-Moser type estimates nor Carleman type estimates unless the system has special structures. In literature, it has been mostly assumed that the systems are regular elliptic and bounndedness of solutions are known in the study of the direct problem. Many inverse problems for the parameter identification use Carleman's estimates which are quite difficult to deal with, because the equations are coupled in the highest derivatives terms. Until now, only partial results were available in the literature concerning the Carleman estimates for strongly coupled systems. Different from the existing methods dealing with weakly or strongly coupled system, such as Fan and Chen [20] and Bellassoued \& Yamamoto [8]. Carleman estimates for a weakly coupled parabolic system could be derived by adding up all Carleman estimates corresponding to each equation. The weakly coupled terms are absorbed by the terms on the left-hand side of the obtained inequality in this process, see Benabdallah et al. [9], Cristofol et al. [17]. As for a strongly coupled parabolic or hyperbolic system, the method above does not work, because the strongly coupled terms could not be simultaneously absorbed by adding up each Carleman estimate. However, to our best knowledge, if the matrix $\left(a_{i, j}\right)_{1 \leq i, j \leq 2}$ formed by the parameters $a_{1,1}, a_{2,2}$ describing the self diffusion of the state variables and $a_{1,2}, a_{2,1}$ describing the influence of each component on another, is diagonalizable then the transformed system satisfies a weakly coupled system. In this case, Carleman estimate is obtained similarly to that of the weakly coupled system, see $[8,21,50]$. But due to the fact that the anisotropy in the bidomain 
equations depends on the fiber directions, and the fact that the fiber direction are space dependent, one cannot diagonalize the problem as in $[8,50]$.

In this paper, we study the stability of the inverse problem of identification of these conductances parameters for a bidomain model: the reconstruction of these conductances from the measurement of electrical potentials over a part of the space boundary during the time and the state variables at a suitable time $t_{0}$. The stability results is based on a global Carleman estimate for a non-linear coupled system with one observation. Our inverse stability results are new because the bidomain system contains a strong coupling term. The technics we shall discuss are similar to the framework using Carleman estimates for inverse problems but the obtained estimates differs from those of $[17,44,53]$ because of the strongly coupled terms. We use the same strategy introduced by $\mathrm{Wu}$ and $\mathrm{Yu}$ in [51] to convert the strongly coupled terms to the gradient of the extra-cellular potential $u_{e}$. Then, we consider the new version of the bidomain model as a whole to establish Carleman estimate under a weaker coupled equations. The paper is organized as follows: In Section 2, we briefly recall the general structure of cardiac cellular membrane models describing the transmembrane potential and the ionic exchange at the cell membrane. Then, we present the bidomain model describing the electrical wave propagation and recall some existence and uniqueness results that have been shown in [49] and we deal with a regularity results of the bidomain solution which will be useful in stability analysis. In section 3, we announce the main stability result including the conditions we need for the identification of multiple parameters. The proof of the main result is divided into two sections. In Section 4, we prove the global Carleman inequality for the reaction-diffusion system. Most of the non-classical parts of the proof of the main result are presented in Section 5 where we prove the stability estimate of conductances parameters.

\section{Mathematical Model}

Let $\Omega \subset \mathbb{R}^{n}(n \geq 1)$ be a bounded connected open set whose boundary $\Gamma=\partial \Omega$ is regular enough, $\left(\Omega \subset \mathbb{R}^{3}\right.$ being the natural domain of the hearth). Let $T>0$ and $\Gamma_{0} \subset \Gamma$ be a relatively open subset of $\Gamma$. We will use the notation $Q=\Omega \times(0, T), \Sigma=\Gamma \times(0, T)$ and $\Sigma_{0}=\Gamma_{0} \times(0, T)$.

We introduce a two reaction-diffusion parabolic PDEs system called bidomain model, coupled to a system of ODEs. This model was proposed in the late 1970s by Tung [47] and is now the generally accepted model of electrical behaviour of cardiac tissue (see Henriquez [24], Keener and Sneyd [31]), can be written as:

$$
\begin{cases}c_{m} \partial_{t} v+I_{i o n}(\bar{\varrho}, v, \mathbf{w}, \mathbf{z})=\operatorname{div}\left(\boldsymbol{\sigma}_{i} \nabla u_{i}\right)+I_{i}^{s} & \text { in } Q, \\ c_{m} \partial_{t} v+I_{i o n}(\bar{\varrho}, v, \mathbf{w}, \mathbf{z})=-\operatorname{div}\left(\boldsymbol{\sigma}_{e} \nabla u_{e}\right)-I_{e}^{s} & \text { in } Q, \\ \partial_{t} \mathbf{w}=\boldsymbol{F}(v, \mathbf{w}) & \text { in } Q, \\ \partial_{t} \mathbf{z}=\boldsymbol{G}(\bar{\varrho}, v, \mathbf{w}, \mathbf{z}) & \text { in } Q, \\ \boldsymbol{\sigma}_{i} \nabla u_{i} \cdot \nu=\boldsymbol{\sigma}_{e} \nabla u_{e} \cdot \nu=0 & \text { on } \Sigma,\end{cases}
$$

where $u_{i}=u_{i}(x, t)$ and $u_{e}=u_{e}(x, t)$ represent, respectively, the intracellular and extracellular electric potentials, whose difference $v:=u_{i}-u_{e}$ is the transmembrane potential. $\mathbf{w}:=\mathbf{w}(x, t)=\left(w_{1}, \ldots, w_{k}\right)(x, t)$ and $\mathbf{z}:=\mathbf{z}(x, t)=\left(z_{1}, \ldots, z_{m}\right)(x, t)$ are the gating variables and the ionic intracellular concentration variables, respectively. The surface capacitance of the membrane is represented by the constant $c_{m}>0$. For simplicity, we shall suppose $c_{m}=1$. Here, $I_{i}^{s}:=I_{i}^{s}(x, t)$ and $I_{e}^{s}:=I_{e}^{s}(x, t)$ are respectively the internal and the external applied current sources.

In an isolated heart, no current flows out of the heart, as expressed by the Neumann conditions. We complete this model with initial data:

$$
v(\cdot, 0)=v_{0}, \quad \mathbf{w}(\cdot, 0)=\mathbf{w}_{0}, \quad \mathbf{z}(\cdot, 0)=\mathbf{z}_{0}, \quad x \in \Omega .
$$

The ionic current $I_{i o n}:=I_{i o n}(x, t)$ and the functions $\boldsymbol{F}$ and $\boldsymbol{G}$ depends of the considering ionic model. 
In the membrane model, the ionic current $I_{i o n}$ has the following general structure [43]:

$$
I_{\text {ion }}(\bar{\varrho}, v, \mathbf{w}, \mathbf{z})=\sum_{i=1}^{N} \bar{\varrho}_{i} y_{i}(v) \prod_{j=1}^{k} w_{j}^{p_{j, i}}\left(v-E_{i}(\mathbf{z})\right)
$$

where $N$ is the number of ionic currents, $\bar{\varrho}_{i}:=\varrho_{i}(x)$ is the maximal conductance associated with the $i^{t h}$ current, $y_{i}$ is a gating function depending only on the membrane potentiel $v, p_{j, i}$ are positive integers exponents and $E_{i}$ is the reversal potential for the $i^{t h}$ current $I_{i}$, which is the related equilibrium (Nernst) potential and is given by

$$
E_{i}(\mathbf{z})=\bar{\gamma}_{i} \log \left(\frac{z_{e}}{z_{i}}\right), \quad \mathbf{z}=\left(z_{1}, \ldots, z_{m}\right)
$$

where $\bar{\gamma}_{i}$ is a constant and $z_{i}, i=1, \ldots, m$, are the intracellular concentrations. The constant $z_{e}$ denotes an extracellular concentration. The anisotropic properties of the two media are modeled by an intracellular and extracellular conductivity tensors $\boldsymbol{\sigma}_{i}(x)$ and $\boldsymbol{\sigma}_{e}(x)$. Generally, the conductivities $\boldsymbol{\sigma}_{i}$ and $\boldsymbol{\sigma}_{e}$ are two matrices given by

$$
\boldsymbol{\sigma}_{j}(x)=\sigma_{j}^{t}(x) \boldsymbol{I}+\left(\sigma_{j}^{l}(x)-\sigma_{j}^{t}(x)\right) \boldsymbol{a}_{l}(x) \boldsymbol{a}_{l}^{T}(x),
$$

where $\sigma_{j}^{l}$ and $\sigma_{j}^{t}, j \in\{i, e\}$ are the intra- and extracellular conductivities along and transversal to the direction of the fiber (parallel to $\boldsymbol{a}_{l}(x)$ ), respectively. In the case of equal anisotropy, i.e the so-called anisotropy ratios $\sigma_{i}^{t} / \sigma_{i}^{l}=\sigma_{e}^{t} / \sigma_{e}^{l}=1$, the $\boldsymbol{\sigma}_{i}$ and $\boldsymbol{\sigma}_{e}$ are simplified as $\boldsymbol{\sigma}_{i}(x)=\sigma_{i}(x) \boldsymbol{I}$ and $\boldsymbol{\sigma}_{e}(x)=\sigma_{e}(x) \boldsymbol{I}$ with $\sigma_{i}(x)=\sigma_{i}^{t}(x)$, $\sigma_{e}(x)=\sigma_{e}^{t}(x)$, which is the case we discussed.

For the ODEs, the dynamics of the gating variable $\mathbf{w}$ is described in the Hodgkin-Huxley formalism by a system of ordinary differential equations which when $w_{j}$ is a gating variable $\left(0 \leq w_{j} \leq 1\right)$ are governed by the following equation,

$$
\partial_{t} w_{j}=F_{j}\left(v, w_{j}\right):=\alpha_{j}(v)\left(1-w_{j}\right)-\beta_{j}(v) w_{j}, \quad j=1, \ldots, k,
$$

where $\alpha_{j}$ and $\beta_{j}$ are positive rational functions of exponentials in $v$. A general expression for both $\alpha_{j}$ and $\beta_{j}$ is given by

$$
\frac{\mu_{1} e^{\mu_{2}\left(v-v_{n}\right)}+\mu_{3}\left(v-v_{n}\right)}{1+\mu_{4} e^{\mu_{5}\left(v-v_{n}\right)}}
$$

where $\mu_{1}, \mu_{3}, \mu_{4}, v_{n}$ are non-negative constants and $\mu_{2}, \mu_{5}$ are positive constants.

The dynamics of the ionic concentration variables $\mathbf{z}$ is described by the additional system of ODEs:

$$
\partial_{t} z_{i}=G_{i}(\bar{\varrho}, v, \mathbf{w}, \mathbf{z}):=-J_{i}\left(\bar{\varrho}, v, \mathbf{w}, \log z_{i}\right)+H_{i}(\bar{\varrho}, v, \mathbf{w}, \mathbf{z}), \quad i=1, \ldots, m .
$$

Assumptions 2.1. We assume that:

1) The conductivities of the intracellular and extracellular $\sigma_{\mathrm{i}}, \sigma_{\mathrm{e}} \in \mathcal{C}^{2}(\bar{\Omega})$, such that $0<\underline{\sigma_{j}}<\sigma_{j}(x)<\overline{\sigma_{j}}$, $j \in\{i, e\}$, in $\bar{\Omega}$.

2) We use the regularized form of the variable $y_{i}(v)$ in hyperbolic functions introduced in [19]. In this case, $y_{i}(v)$ is a $\mathcal{C}^{\infty}$ function with respect to the variable $v$ for $i=1, \ldots, N$, and then is locally Lipschitz since $v$ is bounded, similarly for the function $y_{i}(v) v$.

3) $J_{i}$ and $H_{i}$ are locally Lipschitz continuous functions where:

$$
J_{i} \in \mathcal{C}^{2}\left(\mathbb{R}_{+}^{*} \times \mathbb{R} \times \mathbb{R}^{k} \times \mathbb{R}\right), \quad 0<g_{*}(\mathbf{w}) \leq \frac{\partial J_{i}}{\partial \tau}(\varrho, v, \mathbf{w}, \tau) \leq g^{*}(\mathbf{w}), \quad\left|\frac{\partial J_{i}}{\partial v}(\bar{\varrho}, v, \mathbf{w}, 0)\right| \leq L_{v}(\mathbf{w}),
$$

$g_{*}, g^{*}, L_{v}$ belong to $\mathcal{C}^{1}\left(\mathbb{R}^{k}, \mathbb{R}_{+}\right)$, and

$$
H_{i} \in \mathcal{C}^{2}\left(\mathbb{R}_{+}^{*} \times \mathbb{R} \times \mathbb{R}^{k} \times(0,+\infty)^{m}\right) \cap \operatorname{Lip}\left(\mathbb{R}_{+}^{*} \times \mathbb{R} \times[0,1]^{k} \times(0,+\infty)^{m}\right) .
$$


Remark 2.2. One could find in the literature many refined models based on Hodgkin-Huxley formalism taking into account different quantities. For example, we recall here the following models: Beeler-Reuter $([5], N=$ $4, k=6, m=1)$, phase-I Luo-Rudy ([36], $N=6, k=6, m=1)$, phase-II Luo-Rudy ( [37], $N=10, k=6, m=$ $5)$.

Now, we propose a new approach to the bidomain equations (2.1). Our idea is to use a reformulation of (2.1) as a parabolic PDE coupled to an elliptic one, by replacing $u_{i}=v+u_{e}$ in the first PDE. The boundary condition is also reformulated in terms of $v$ and $u_{e}$. We have:

$$
\begin{cases}\partial_{t} v-\operatorname{div}\left(\sigma_{i} \nabla v\right)+I_{i o n}(\bar{\varrho}, v, \mathbf{w}, \mathbf{z})=\operatorname{div}\left(\sigma_{i} \nabla u_{e}\right)+I_{i}^{s} & \text { in } Q, \\ -\operatorname{div}\left(\sigma_{e} \nabla u_{e}\right)-I_{i o n}(\bar{\varrho}, v, \mathbf{w}, \mathbf{z})=\partial_{t} v+I_{e}^{s} & \text { in } Q \\ \partial_{t} \mathbf{w}=\boldsymbol{F}(v, \mathbf{w}) & \text { in } Q \\ \partial_{t} \mathbf{z}=\boldsymbol{G}(\bar{\varrho}, v, \mathbf{w}, \mathbf{z}) & \text { in } Q \\ \sigma_{i} \nabla v \cdot \nu+\sigma_{i} \nabla u_{e} \cdot \nu=0 & \text { on } \Sigma, \\ \sigma_{e} \nabla u_{e} \cdot \nu=0 & \text { on } \Sigma,\end{cases}
$$

By using the second equation in (2.10), we obtain

$$
\begin{aligned}
-\operatorname{div}\left(\sigma_{i} \nabla u_{e}\right) & =-\nabla \cdot\left(\sigma_{i} \sigma_{e}^{-1} \sigma_{e} \nabla u_{e}\right) \\
& =-\nabla\left(\sigma_{i} \sigma_{e}^{-1}\right) \cdot \sigma_{e} \nabla u_{e}-\sigma_{i} \sigma_{e}^{-1} \operatorname{div}\left(\sigma_{e} \nabla u_{e}\right) \\
& =\sigma_{e}^{-1}\left(\sigma_{i} \partial_{t} v+\sigma_{i}\left(I_{\text {ion }}+I_{e}^{s}\right)\right)+\boldsymbol{A}(x) \cdot \nabla u_{e}
\end{aligned}
$$

with

Substituting (2.11) into (2.10) yields

$$
\boldsymbol{A}(x):=-\nabla\left(\sigma_{e}^{-1} \sigma_{i}\right) \sigma_{e}=\sigma_{e}^{-1}\left(\sigma_{i} \nabla \sigma_{e}-\sigma_{e} \nabla \sigma_{i}\right)
$$

$$
\left\{\begin{array}{lr}
\left(1+\sigma_{i} \sigma_{e}^{-1}\right) \partial_{t} v-\operatorname{div}\left(\sigma_{i} \nabla v\right)=-\left(1+\sigma_{i} \sigma_{e}^{-1}\right) I_{i o n}(\bar{\varrho}, v, \mathbf{w}, \mathbf{z})-\boldsymbol{A}(x) \cdot \nabla u_{e}+I_{i}^{s}-\sigma_{i} \sigma_{e}^{-1} I_{e}^{s} & \text { in } Q \\
-\operatorname{div}\left(\sigma_{e} \nabla u_{e}\right)=\partial_{t} v+I_{i o n}(\bar{\varrho}, v, \mathbf{w}, \mathbf{z})+I_{e}^{s} & \text { in } Q \\
\partial_{t} \mathbf{w}=\boldsymbol{F}(v, \mathbf{w}) & \text { in } Q \\
\partial_{t} \mathbf{z}=\boldsymbol{G}(\bar{\varrho}, v, \mathbf{w}, \mathbf{z}) & \text { in } Q \\
\sigma_{i} \nabla v \cdot \nu+\sigma_{i} \nabla u_{e} \cdot \nu=0 & \text { on } \Sigma, \\
\sigma_{e} \nabla u_{e} \cdot \nu=0 & \text { on } \Sigma,
\end{array}\right.
$$

Hence we consider the problem of finding unknown functions $v, u_{e}, \mathbf{w}$ and $\mathbf{z}$ verifying $(2.13)$. Since $v=u_{i}-u_{e}$, it is natural decompose the initial condition $v_{0}$ as $v_{0}=u_{i, 0}-u_{e, 0}$.

Now, we state some regularity and estimate results proved in $[48,49]$ of a strong solution to problem (2.1).

Lemma 2.3. Assume that $\left(v_{0}, \mathbf{w}_{0}, \mathbf{z}_{0}\right) \in H^{2}(\Omega) \times L^{2}(\Omega)^{k} \times L^{2}(\Omega)^{m}$, with $\log \mathbf{z}_{0}:=\left(\log z_{0,1}, \ldots, \log z_{0, m}\right) \in$ $L^{2}(\Omega)^{m}$, and

then we have

$$
I_{i, e}^{s} \in L^{p}\left(0, T ; L^{2}(\Omega)\right) \cap H^{2}\left(0, T ; H^{2}(\Omega)\right), p>4,
$$

1)

$$
u_{i, e} \in L^{p}\left(0, T ; H^{2}(\Omega)\right),
$$




$$
\begin{gathered}
v \in W^{1, p}\left(0, T ; L^{2}(\Omega)\right) \cap L^{2}\left(0, T ; H^{2}(\Omega)\right) \cap \mathcal{C}^{0}\left([0, T], \mathcal{C}^{0}(\Omega)\right), \quad \text { for } p>4, \\
\mathbf{w}: Q \rightarrow[0,1]^{k} \text { measurable, } \quad \mathbf{z}: Q \rightarrow(0,+\infty)^{m} \quad \text { measurable, } \\
w_{j}(x, .) \in \mathcal{C}^{1}(0, T) \cap \mathcal{C}^{0}([0, T]), \quad \text { for a.e. }, \quad x \in \Omega, \quad j=1, \ldots, k, \\
z_{i}(x, .) \in \mathcal{C}^{1}(0, T) \cap \mathcal{C}^{0}([0, T]), \quad \text { for a.e., } \quad x \in \Omega, \quad i=1, \ldots, m, \\
\mathbf{z} \in H^{1}\left(0, T ; L^{2}(\Omega)\right)^{m} \cap L^{\infty}(Q)^{m}, \quad \log \mathbf{z}:=\left(\log z_{1}, \ldots, \log z_{m}\right) \in L^{\infty}(Q)^{m} .
\end{gathered}
$$

2) There exists a constant $C>0$, independant of $v, \mathbf{w}, \mathbf{z}$, such that

$$
|\mathbf{z}(x, t)| \leq C\left(1+\left|\mathbf{z}_{0}(x)\right|+\|v(x)\|_{L^{2}(0, t)}\right), \quad \text { a.e., } x \in \Omega,
$$

and

$$
|\log \mathbf{z}(x, t)|+\left|\partial_{t} \mathbf{z}(x, t)\right| \leq C\left(1+\left|\mathbf{z}_{0}(x)\right|+\|v(x)\|_{\mathcal{C}^{0}(0, t)}\right), \quad \text { a.e., } x \in \Omega,
$$

$\forall t \in[0, T]$, for a.e. $x \in \Omega$.

3) There exists a constant $C>0$, depending on $m, T$ such that, $\forall i=1, \ldots, m$,

$$
z_{i}(x, t) \geq \exp \left[-C\left(1+\left\|\mathbf{z}_{0}\right\|_{L^{\infty}}+\|v\|_{\mathcal{C}^{0}\left([0, T], \mathcal{C}^{0}(\Omega)\right)}\right)\right]>0, \quad \forall t \in[0, T], \text { a.e., } x \in \Omega .
$$

4) There exists $M_{\infty}>0$, depending on the data of the problem, such that:

$$
\sup \{|v(x, t)|:(x, t) \in Q\} \leq M_{\infty} .
$$

Now, we will establish regularity results for the solution of the bidomain system (2.13). The aim is to improve the regularity results given in Lemma 2.3 in order to satisfy some assumptions useful for the stability result.

Proposition 2.4. Let $\left(v, u_{e}, \mathbf{w}, \mathbf{z}\right)$ be the solution of bidomain system (2.1), with initial conditions $\left(v_{0}, u_{e, 0}, \mathbf{w}_{0}, \mathbf{z}_{0}\right)$

- If $v_{0}, u_{e, 0} \in H^{2}(\Omega), \mathbf{w}_{0} \in L^{2}(\Omega)^{k}, \mathbf{z}_{0} \in L^{2}(\Omega)^{m}$, and $I_{i, e}^{s}$ verify the regularity (2.14), then

$$
\begin{gathered}
v, u_{e} \in W^{1, \infty}\left(0, T ; H^{1}(\Omega)\right) \cap L^{2}\left(0, T ; H^{2}(\Omega)\right) \cap H^{1}\left(0, T ; H^{1}(\Omega)\right), \\
\mathbf{w} \in W^{1, \infty}\left(0, T ; L^{2}(\Omega)\right)^{k}, \quad \text { and } \quad \mathbf{z} \in W^{1, \infty}\left(0, T ; L^{2}(\Omega)\right)^{m} .
\end{gathered}
$$

Moreover if

then

$$
\mathbf{w}_{0} \in H^{1}(\Omega)^{k}, \quad \text { and } \quad \mathbf{z}_{0} \in H^{1}(\Omega)^{m},
$$

$$
\mathbf{w} \in W^{1, \infty}\left(0, T ; H^{1}(\Omega)\right)^{k}, \quad \text { and } \quad \mathbf{z} \in W^{1, \infty}\left(0, T ; H^{1}(\Omega)\right)^{m} .
$$

- If $v_{0}, u_{e, 0} \in H^{4}(\Omega), \mathbf{w}_{0} \in H^{2}(\Omega)^{k}, \mathbf{z}_{0} \in H^{2}(\Omega)^{m}$, and $I_{i, e}^{s}$ verify the regularity (2.14). Then the solution of (2.1) satisfies

$$
\begin{gathered}
v, u_{e} \in H^{1}\left(0, T ; H^{3}(\Omega)\right) \cap H^{2}\left(0, T ; H^{1}(\Omega)\right), \\
\mathbf{w} \in W^{1, \infty}\left(0, T ; H^{2}(\Omega)\right)^{k} \cap H^{2}\left(0, T ; L^{2}(\Omega)\right)^{k}, \\
\mathbf{z} \in W^{1, \infty}\left(0, T ; H^{2}(\Omega)\right)^{m} \cap H^{2}\left(0, T ; L^{2}(\Omega)\right)^{m} .
\end{gathered}
$$

Moreover if

$$
\mathbf{w}_{0} \in H^{3}(\Omega)^{k}, \quad \text { and } \quad \mathbf{z}_{0} \in H^{3}(\Omega)^{m},
$$

then

$$
\begin{gathered}
\mathbf{w} \in W^{1, \infty}\left(0, T ; H^{3}(\Omega)\right)^{k} \hookrightarrow H^{1}\left(0, T ; H^{3}(\Omega)\right)^{k}, \\
\mathbf{z} \in W^{1, \infty}\left(0, T ; H^{3}(\Omega)\right)^{m} \hookrightarrow H^{1}\left(0, T ; H^{3}(\Omega)\right)^{m} .
\end{gathered}
$$

The proof of proposition 2.4 is provided in the Appendix 7 . 


\section{InVERSE PROBlem: MAIN RESUlt}

Our inverse problem is related to determination of multiple ionic parameter $\bar{\varrho}_{i}, 1 \leq i \leq N$, of a non linear parabolic reaction diffusion system coupled with an ordinary differential equations from a set of boundary observations $\left(u_{e, \ell}, \mathbf{w}_{\ell}, \mathbf{z}_{\ell}\right)_{\mid \Gamma_{0} \times(0, T)}$ and $\left(u_{e, \ell}\left(x, t_{0}\right), \mathbf{w}_{\ell}\left(x, t_{0}\right), \mathbf{z}_{\ell}\left(x, t_{0}\right)\right), x \in \Omega, 1 \leq \ell \leq N$.

In order to formulate our results, we need to introduce the following notations: For a sequence functions $\left(\widetilde{v}_{\ell}, \widetilde{\mathbf{w}}_{\ell}, \widetilde{\mathbf{z}}_{\ell}\right) \in H^{3}(\Omega) \times \mathcal{C}^{1}(\Omega)^{k} \times \mathcal{C}^{1}(\Omega)^{m}$, we define the $N \times N$ matrix $\Lambda$ as follows

$$
\Lambda\left(\widetilde{v}_{\ell}(x), \widetilde{\mathbf{w}}_{\ell}(x), \widetilde{\mathbf{z}}_{\ell}(x)\right)=\left(\begin{array}{cccc}
S_{1,1}(x) & S_{2,1}(x) & \ldots & S_{N, 1}(x) \\
S_{1,2}(x) & S_{2,2}(x) & \ldots & S_{N, 2}(x) \\
\vdots & \vdots & \ddots & \vdots \\
S_{1, N}(x) & S_{2, N}(x) & \ldots & S_{N, N}(x)
\end{array}\right)
$$

where

$$
S_{i, \ell}(x)=y_{i}\left(\widetilde{v}_{\ell}(x)\right)\left(\widetilde{v}_{\ell}(x)-E_{i}\left(\widetilde{\mathbf{z}}_{\ell}(x)\right)\right) \prod_{j=1}^{k}\left(\widetilde{w}_{\ell}\right)_{j}^{p_{j, i}}(x), 1 \leq \ell, i \leq N .
$$

Let us fix constant $M_{0}>0$. We introduce an admissible set of unknown coefficients vector $\bar{\varrho}$ by

$$
\mathcal{A}=\left\{\bar{\varrho} \in H^{3}(\Omega)^{N},\|\bar{\varrho}\|_{l^{2}\left(H^{3}(\Omega)^{N}\right)} \leq M_{0}\right\} .
$$

We obtain the following stability result.

Theorem 3.1. Let $t_{0} \in(0, T), \Gamma_{0}$ be a subdomain of $\partial \Omega$ and let $\bar{\varrho}^{(2)} \in \mathcal{A}$ be arbitrary fixed. We assume that $I_{i, e}^{s, \ell} \in L^{p}\left(0, T ; L^{2}(\Omega)\right) \cap H^{1}\left(0, T ; H^{2}(\Omega)\right), p>4,1 \leq \ell \leq N$, satisfy

$$
\operatorname{det}\left(\Lambda\left(v_{\ell}^{(2)}\left(x, t_{0}\right), \mathbf{w}_{\ell}^{(2)}\left(x, t_{0}\right), \mathbf{z}_{\ell}^{(2)}\left(x, t_{0}\right)\right)\right) \neq 0, \quad \forall x \in \Omega \text {. }
$$

Here $v_{\ell}^{(2)}, \mathbf{w}_{\ell}^{(2)}$ and $\mathbf{z}_{\ell}^{(2)}$ are the state variables of (2.13) with $\bar{\varrho}=\bar{\varrho}^{(2)}$ and $I_{i, e}^{s}=I_{i, e}^{s, \ell}$. Furthermore, we assume that

$$
\left\|v_{\ell}^{(2)}\right\|_{\mathcal{C}^{0}\left([0, T] ; \mathcal{C}^{1}(\bar{\Omega})\right)}+\left\|\mathbf{w}_{\ell}^{(2)}\right\|_{\mathcal{C}^{0}\left([0, T] ; \mathcal{C}^{1}(\bar{\Omega})\right)^{k}}+\left\|\mathbf{z}_{\ell}^{(2)}\right\|_{\mathcal{C}^{0}\left([0, T] ; \mathcal{C}^{1}(\bar{\Omega})\right)^{m}} \leq M,
$$

for some positive $M$. Then there exists a constant $C>0$ such that we have:

$$
\begin{aligned}
\left\|\varrho^{(1)}-\bar{\varrho}^{(2)}\right\|_{l^{2}\left(L^{2}(\Omega)\right)^{N}} & \leq C \sum_{\ell=1}^{N}\left(\left\|\left(u_{e, \ell}^{(1)}-u_{e, \ell}^{(2)}\right)\right\|_{H^{2}\left(0, T ; H^{1}\left(\Gamma_{0}\right)\right)}\right. \\
& \left.+\left\|\left(u_{e, \ell}^{(1)}-u_{e, \ell}^{(2)}\right)\left(\cdot, t_{0}\right)\right\|_{H^{2}(\Omega)}+\left\|\left(\mathbf{w}_{\ell}^{(1)}-\mathbf{w}_{\ell}^{(2)}\right)\left(\cdot, t_{0}\right)\right\|_{L^{2}(\Omega)^{k}}+\left\|\left(\mathbf{z}_{\ell}^{(1)}-\mathbf{z}_{\ell}^{(2)}\right)\left(\cdot, t_{0}\right)\right\|_{L^{2}(\Omega)^{m}}\right)
\end{aligned}
$$

for all $\bar{\varrho}^{(1)} \in \mathcal{A}$.

Remark 3.2. The condition (3.4) is a straightforward consequence of the Proposition 2.4 and a Sobolev embedding theorem (e.g., Thm. 5.4 in [3]).

$$
H^{1}\left(0, T ; H^{3}(\Omega)\right) \hookrightarrow \mathcal{C}^{0}\left([0, T] ; \mathcal{C}^{1}(\bar{\Omega})\right),
$$

if the initial conditions verify

$$
v_{\ell}^{(2)}(t=0) \in H^{4}(\Omega), \quad u_{e, \ell}^{(2)}(t=0) \in H^{4}(\Omega), \quad \mathbf{w}_{\ell}^{(2)}(t=0) \in H^{3}(\Omega)^{k}, \quad \text { and } \quad \mathbf{z}_{\ell}^{(2)}(t=0) \in H^{3}(\Omega)^{m} .
$$

We introduced the condition (3.4), because it may be obtained with a less regularities on the initial conditions. 
By Theorem 3.1, we can readily derive the uniqueness in the inverse problem.

Corollary 3.3. Under the same assumptions as in Theorem 3.1 and if

$$
\begin{gathered}
\left(u_{e, \ell}^{(1)}\left(x, t_{0}\right), \mathbf{w}_{\ell}^{(1)}\left(x, t_{0}\right), \mathbf{z}_{\ell}^{(1)}\left(x, t_{0}\right)\right)=\left(u_{e, \ell}^{(2)}\left(x, t_{0}\right), \mathbf{w}_{\ell}^{(2)}\left(x, t_{0}\right), \mathbf{z}_{\ell}^{(2)}\left(x, t_{0}\right)\right), \quad \text { in } x \in \Omega, \\
u_{e, \ell}^{(1)}(x, t)=u_{e, \ell}^{(2)}(x, t), \quad \text { in } \Gamma_{0} \times(0, T),
\end{gathered}
$$

for $\ell=1, \ldots, N$, then $\bar{\varrho}^{(1)}=\bar{\varrho}^{(2)}$ in $\Omega$.

Since the number of the unknown coefficients is $N$, it is natural to expect that $N$-times observations can yield the Lipschitz stability. Our tool are a weighted $L^{2}$-norm estimates of the solution of a PDE called Carleman estimates, where the weight takes an exponential form. They are an important tool in subjects in analysis of PDEs such as unique continuation $[13,26]$, control theory $[15,18]$ and coefficient inverse problems $[4,6,7,27-29,32,52]$.

\section{Global Carleman inequality for Reaction-Diffusion System}

In this section, we give Carleman estimate for the reaction-diffusion model with a finite observations acting on a subboundary $\Sigma_{0}$ of $\partial \Omega$ on the right-hand side of the estimate. This Carleman estimate would be used later for the stability and uniqueness of the solution of the parameter identification problem. We are interested in identifying the parameters $\bar{\varrho}_{i}, i=1, \ldots, N$, where $\bar{\varrho}_{i}$ is the maximal conductance associated with the $i^{\text {th }}$ current.

In order to frame a Carleman type estimate, we shall first introduce a particular type of weight functions.

\subsection{Weight functions and Carleman estimate}

For our Carleman estimate, we need a weight function $\beta$ with the following properties [18].

Lemma 4.1. Let $\Gamma_{0} \neq \emptyset \subset \partial \Omega$ be an arbitrary relatively open subset. Then there exists a function $\beta \in \mathcal{C}^{2}(\bar{\Omega})$ such that

$$
\beta(x)>0 \quad \forall x \in \Omega, \quad|\nabla \beta(x)|>0 \quad \forall x \in \bar{\Omega}, \quad \text { and } \quad \boldsymbol{\sigma}_{i, e}(x) \nabla \beta(x) \cdot \nu(x) \leq 0, \quad x \in \partial \Omega \backslash \Gamma_{0} .
$$

Proof 4.1. The proof of the Lemma 4.1 is given in [27].

We set

$$
\varphi(x, t)=\frac{e^{\lambda \beta(x)}}{t(T-t)}
$$

and

$$
\eta(x, t)=\frac{e^{2 \lambda\|\beta\|_{\infty}}-e^{\lambda \beta(x)}}{t(T-t)}
$$

where $\lambda>0$ and $t \in(0, T)$. Let us consider a boundary value problem for the parabolic operator:

$$
\begin{cases}\mathfrak{L} u(x, t) \equiv \partial_{t} u-\sum_{i, j=1}^{n} \sigma_{i j}(x) \partial_{i} \partial_{j} u=\mathcal{F}, & \text { in } \mathrm{Q}, \\ \nabla u . \nu=0, & \text { on } \Sigma .\end{cases}
$$

We recall that

$$
H^{2,1}(Q)=\left\{u \in L^{2}(Q) \mid \partial_{t} u, \partial_{i} u, \partial_{i} \partial_{j} u \in L^{2}(Q), 1 \leq i, j \leq n\right\}
$$


Moreover, we set

$$
\tilde{\gamma}=\sum_{i, j=1}^{n}\left\|\sigma_{i j}\right\|_{C^{2}(\bar{\Omega})},
$$

By $\beta$ constructed in Lemma 4.1, we have the following Lemma:

Lemma 4.2. Let $\sigma(x) \in C^{2}(\bar{\Omega})$ and the function $\varphi, \eta$ be defined by (4.1)-(4.3). Then there exists a number $\lambda_{0}>0$ such that for an arbitrary $\lambda \geq \lambda_{0}$, we can choose a constant $s_{0}(\lambda) \geq 0$ satisfying: there exists a constant $C=C\left(s_{0}, \lambda_{0}\right)>0$ such that:

$$
\begin{aligned}
& \int_{Q}\left\{(s \varphi)^{p-1}\left(\left|\partial_{t} u\right|^{2}+\sum_{i, j=1}^{n}\left|\partial_{i} \partial_{j} u\right|^{2}\right)+(s \varphi)^{p+1} \lambda^{2}|\nabla u|^{2}+(s \varphi)^{p+3} \lambda^{4}|u|^{2}\right\} e^{-2 s \eta} d x d t \\
& \quad \leq C \int_{Q}(s \varphi)^{p}|\mathfrak{L} u|^{2} e^{-2 s \eta} d x d t+C e^{C(\lambda) s} \int_{\Sigma_{0}}\left(\left|\partial_{t} u\right|^{2}+|\nabla u|^{2}+|u|^{2}\right) d S d t
\end{aligned}
$$

for all $s>s_{0}, p=0,1,2$, and all $u \in H^{2,1}(Q)$. Here the constant $C>0$ depends continuously on $\lambda_{0}$ but is independent of $s$, and $\lambda_{0}$ depends continuously on $\tilde{\gamma}$.

Proof 4.2. The proof of this lemma is similar to the proof given in [14]. We can further refer to [23].

\subsection{Global Carleman inequality for bidomain equations}

We consider the solutions $\left(v^{(n)}, u_{e}^{(n)}, \mathbf{w}^{(n)}, \mathbf{z}^{(n)}\right), n=1,2$, to the following systems

$$
\left\{\begin{array}{lr}
\left(1+\sigma_{i} \sigma_{e}^{-1}\right) \partial_{t} v^{(n)}-\operatorname{div}\left(\sigma_{i} \nabla v^{(n)}\right)+\left(1+\sigma_{i} \sigma_{e}^{-1}\right) I_{i o n}\left(v^{(n)}, \mathbf{w}^{(n)}, \mathbf{z}^{(n)}\right)= & \text { in } Q, \\
\boldsymbol{A}(x) \cdot \nabla u_{e}^{(n)}+I_{i}^{s}-\sigma_{i} \sigma_{e}^{-1} I_{e}^{s} & \text { in } Q, \\
-\operatorname{div}\left(\sigma_{e} \nabla u_{e}^{(n)}\right)=I_{i o n}\left(v^{(n)}, \mathbf{w}^{(n)}, \mathbf{z}^{(n)}\right)+\partial_{t} v^{(n)}+I_{e}^{s} & \text { in } Q, \\
\partial_{t} \mathbf{w}^{(n)}=\boldsymbol{F}\left(v^{(n)}, \mathbf{w}^{(n)}\right) & \text { in } Q, \\
\partial_{t} \mathbf{z}^{(n)}=\boldsymbol{G}\left(v^{(n)}, \mathbf{w}^{(n)}, \mathbf{z}^{(n)}\right) & \text { on } \Sigma, \\
\sigma_{i} \nabla v^{(n)} \cdot \nu+\sigma_{i} \nabla u_{e}^{(n)} \cdot \nu=0 & \text { on } \Sigma, \\
\sigma_{e} \nabla u_{e}^{(n)} \cdot \nu=0 &
\end{array}\right.
$$

and we consider the difference

$$
v=v^{(1)}-v^{(2)}, \quad u_{e}=u_{e}^{(1)}-u_{e}^{(2)}, \quad \mathbf{w}=\mathbf{w}^{(1)}-\mathbf{w}^{(2)}, \quad \mathbf{z}=\mathbf{z}^{(1)}-\mathbf{z}^{(2)}, \quad \bar{\varrho}=\bar{\varrho}^{(1)}-\bar{\varrho}^{(2)} .
$$

Then, $\left(v, u_{e}, \mathbf{w}, \mathbf{z}\right)$ is solution to the following problem

$$
\begin{cases}\left(1+\sigma_{i} \sigma_{e}^{-1}\right) \partial_{t} v-\operatorname{div}\left(\sigma_{i} \nabla v\right)=-\left(1+\sigma_{i} \sigma_{e}^{-1}\right) h-\boldsymbol{A}(x) \cdot \nabla u_{e} & \text { in } Q, \\ -\operatorname{div}\left(\sigma_{e} \nabla u_{e}\right)=h+\partial_{t} v & \text { in } Q, \\ \partial_{t} \mathbf{w}=\Phi & \text { in } Q, \\ \partial_{t} \mathbf{z}=\Psi & \text { in } Q, \\ \sigma_{i} \nabla v \cdot \nu+\sigma_{i} \nabla u_{e} \cdot \nu=0 & \text { on } \Sigma, \\ \sigma_{e} \nabla u_{e} \cdot \nu=0 & \text { on } \Sigma,\end{cases}
$$


Here

$$
\begin{aligned}
h:= & =I_{i o n}\left(\bar{\varrho}^{(1)}, v^{(1)}, \mathbf{w}^{(1)}, \mathbf{z}^{(1)}\right)-I_{i o n}\left(\varrho^{(2)}, v^{(2)}, \mathbf{w}^{(2)}, \mathbf{z}^{(2)}\right) \\
& =\mathbf{S}^{\top}\left(v^{(2)}, \mathbf{w}^{(2)}, \mathbf{z}^{(2)}\right) \cdot \bar{\varrho}+R\left(v^{(1)}, \mathbf{w}^{(1)}, \mathbf{z}^{(1)}, v^{(2)}, \mathbf{w}^{(2)}, \mathbf{z}^{(2)}\right),
\end{aligned}
$$

where the vector $\mathbf{S}=\left(S_{i}\right)_{1 \leq i \leq N}$ is defined as follow:

$$
S_{i}\left(v^{(2)}, \mathbf{w}^{(2)}, \mathbf{z}^{(2)}\right)=y_{i}\left(v^{(2)}\right)\left(v^{(2)}-E_{i}\left(\mathbf{z}^{(2)}\right)\right) \prod_{j=1}^{k}\left(w_{j}^{(2)}\right)^{p_{j, i}}, \quad i=1, \ldots, N,
$$

and

$$
R\left(v^{(1)}, \mathbf{w}^{(1)}, \mathbf{z}^{(1)}, v^{(2)}, \mathbf{w}^{(2)}, \mathbf{z}^{(2)}\right)=I_{\text {ion }}\left(\bar{\varrho}^{(2)}, v^{(1)}, \mathbf{w}^{(1)}, \mathbf{z}^{(1)}\right)-I_{\text {ion }}\left(\bar{\varrho}^{(2)}, v^{(2)}, \mathbf{w}^{(2)}, \mathbf{z}^{(2)}\right) .
$$

In (4.11), ${ }^{\top}$ represent the transpose of any matrix and the expression $\mathbf{S}^{\top}\left(v^{(2)}, \mathbf{w}^{(2)}, \mathbf{z}^{(2)}\right) \cdot \bar{\varrho}$ is the Euclidian scalar products of the row vector $\mathbf{S}^{\top}$ and the colon vector $\bar{\varrho}:=\left(\bar{\varrho}_{i}\right)_{1 \leq i \leq N}$ formed by the ionic parameters $\varrho_{i}$. Finally, the functions $\Phi$ and $\Psi$ are respectively given by:

$$
\begin{aligned}
\Phi & =\boldsymbol{F}\left(v^{(1)}, \mathbf{w}^{(1)}\right)-\boldsymbol{F}\left(v^{(2)}, \mathbf{w}^{(2)}\right) \\
\Psi & =\boldsymbol{G}\left(\bar{\varrho}^{(1)}, v^{(1)}, \mathbf{w}^{(1)}, \mathbf{z}^{(1)}\right)-\boldsymbol{G}\left(\bar{\varrho}^{(2)}, v^{(2)}, \mathbf{w}^{(2)}, \mathbf{z}^{(2)}\right) .
\end{aligned}
$$

In the next, we denote

$$
R(x, t)=R\left(v^{(1)}, \mathbf{w}^{(1)}, \mathbf{z}^{(1)}, v^{(2)}, \mathbf{w}^{(2)}, \mathbf{z}^{(2)}\right)(x, t), \quad \mathbf{S}(x, t)=\mathbf{S}^{\top}\left(v^{(2)}, \mathbf{w}^{(2)}, \mathbf{z}^{(2)}\right)(x, t),
$$

and

$$
h(x, t):=\mathbf{S}^{\top}(x, t) \cdot \bar{\varrho}(x)+R(x, t) .
$$

Our Carleman estimate for the bidomain equations (4.10) is as follow.

Lemma 4.3. Let the Assumptions 2.1 be fulfilled and the function $\varphi, \eta$ be defined by (4.1)-(4.3). Then there exists a number $\lambda_{0}>0$ such that for an arbitrary $\lambda \geq \lambda_{0}$, we can choose $s_{0}(\lambda) \geq 0$ such that for each $s \geq s_{0}(\lambda)$, a solution $\left(v, u_{e}\right) \in H^{2,1}(Q)$ of the problem (4.10) satisfies the following inequality:

$$
\begin{aligned}
\int_{Q}\left\{s \varphi \left(\left|\partial_{t} v\right|^{2}+\right.\right. & \left.\sum_{i, j=1}^{n}\left|\partial_{i} \partial_{j} v\right|^{2}\right)+(s \varphi)^{3} \lambda^{2}|\nabla v|^{2} \\
+(s \varphi)^{2} \lambda^{2}\left|\nabla u_{e}\right|^{2}+(s \varphi)^{5} \lambda^{4} \lambda^{4}|v|^{2} & \left.+\sum_{i, j=1}^{n}\left|\partial_{i}\right|^{2}\right\}\left.\partial_{j} u_{e}\right|^{2} \\
& +\tilde{C} \int_{\Sigma_{0}}\left\{\left|\partial_{t} v\right|^{2}+|\nabla v|^{2}+|v|^{2}+\left|\nabla u_{e}\right|^{2}+\left|u_{e}\right|^{2}\right\} d S d t
\end{aligned}
$$

where the constant $C$ depends continuously on $\lambda_{0}$.

Proof 4.3. From the boundary conditions of the Problem (4.10), we can write $\sigma_{i} \nabla v \cdot \nu=0$ on $\Sigma$. Then applying estimate (4.7) with $p=2$ to the solution $v$ of (4.10), we have the estimate

$$
\begin{aligned}
\int_{Q}\left\{s \varphi \left(\left|\partial_{t} v\right|^{2}+\right.\right. & \left.\left.\sum_{i, j=1}^{n}\left|\partial_{i} \partial_{j} v\right|^{2}\right)+(s \varphi)^{3} \lambda^{2}|\nabla v|^{2}+(s \varphi)^{5} \lambda^{4}|v|^{2}\right\} e^{-2 s \eta} d x d t \\
& \leq C \int_{Q}(s \varphi)^{2}\left(\left|\nabla u_{e}\right|^{2}+|h|^{2}\right) e^{-2 s \eta} d x d t+C e^{C(\lambda) s} \int_{\Sigma_{0}}\left\{\left|\partial_{t} v\right|^{2}+|\nabla v|^{2}+|v|^{2}\right\} d S d t .
\end{aligned}
$$


Similarly, applying estimate (4.7) with $p=1$ to the solution $u_{e}$ of (4.10), we get

$$
\begin{aligned}
\int_{Q}\left\{\sum_{i, j=1}^{n}\left|\partial_{i} \partial_{j} u_{e}\right|^{2}+(s \varphi)^{2} \lambda^{2}\left|\nabla u_{e}\right|^{2}+(s \varphi)^{4} \lambda^{4}\left|u_{e}\right|^{2}\right\} e^{-2 s \eta} d x d t & \\
& \leq C \int_{Q}(s \varphi)\left(\left|\partial_{t} v\right|^{2}+|h|^{2}\right) e^{-2 s \eta} d x d t+C e^{C(\lambda) s} \int_{\Sigma_{0}}\left\{\left|\nabla u_{e}\right|^{2}+\left|u_{e}\right|^{2}\right\} d S d t
\end{aligned}
$$

By $\frac{4}{T^{2}} \leq \varphi \leq\left(\frac{T}{2}\right)^{2} \varphi^{2},(2 C) \times(4.19)+(4.20)$, and $\lambda$ sufficiently large, we obtain the Lemma 4.3.

\section{Stability estimate OF CONDUCTANCES PARAMETERS}

This section is devoted to proof Theorem 3.1. Since the proof technically looks very awkward firstly we demonstrate some preliminary lemma.

Lemma 5.1. Under the same hypothesis of Lemma 4.3, we have

$$
\begin{aligned}
& \int_{Q}\left\{\left|\partial_{t}^{2} v\right|^{2}+\sum_{i, j=1}^{n}\left|\partial_{i} \partial_{j} \partial_{t} v\right|^{2}+(s \varphi)^{2} \lambda^{2}\left|\nabla \partial_{t} v\right|^{2}+(s \varphi)^{4} \lambda^{4}\left|\partial_{t} v\right|^{2}+(s \varphi)\left(\left|\partial_{t} v\right|^{2}+\sum_{i, j=1}^{n}\left|\partial_{i} \partial_{j} v\right|^{2}\right)\right. \\
& \quad+(s \varphi)^{3} \lambda^{2}|\nabla v|^{2}+(s \varphi)^{5} \lambda^{4}|v|^{2}+(s \varphi)^{-1} \sum_{i, j=1}^{n}\left|\partial_{i} \partial_{j} \partial_{t} u_{e}\right|^{2}+(s \varphi) \lambda^{2}\left|\nabla \partial_{t} u_{e}\right|^{2}+(s \varphi)^{3} \lambda^{4}\left|\partial_{t} u_{e}\right|^{2} \\
& \left.\quad+\sum_{i, j=1}^{n}\left|\partial_{i} \partial_{j} u_{e}\right|^{2}+(s \varphi)^{2} \lambda^{2}\left|\nabla u_{e}\right|^{2}+(s \varphi)^{4} \lambda^{4}\left|u_{e}\right|^{2}\right\} e^{-2 s \eta} d x d t \leq C \int_{Q}\left(s \varphi\left|\partial_{t} h\right|^{2}+(s \varphi)^{2}|h|^{2}\right) e^{-2 s \eta} d x d t \\
& \quad+\tilde{C} \int_{\Sigma_{0}}\left\{\left|\partial_{t}^{2} v\right|^{2}+\left|\partial_{t} v\right|^{2}+\left|\nabla \partial_{t} v\right|^{2}+|\nabla v|^{2}+|v|^{2}+\left|\nabla \partial_{t} u_{e}\right|^{2}+\left|\partial_{t} u_{e}\right|^{2}+\left|\nabla u_{e}\right|^{2}+\left|u_{e}\right|^{2}\right\} d S d t
\end{aligned}
$$

Proof 5.1. Taking the time derivative of the first equation of (4.10), we see that

$$
\left(1+\sigma_{i} \sigma_{e}^{-1}\right) \partial_{t}^{2} v-\operatorname{div}\left(\sigma_{i} \nabla \partial_{t} v\right)=-\boldsymbol{A}(x) \cdot \nabla \partial_{t} u_{e}-\left(1+\sigma_{i} \sigma_{e}^{-1}\right) \partial_{t} h
$$

Applying Lemma 4.2 with $p=1$ to the solution $\partial_{t} v$ of (5.2), we have the estimate

$$
\begin{aligned}
\int_{Q}\left\{\left|\partial_{t}^{2} v\right|^{2}\right. & \left.+\sum_{i, j=1}^{n}\left|\partial_{i} \partial_{j} \partial_{t} v\right|^{2}+(s \varphi)^{2} \lambda^{2}\left|\nabla \partial_{t} v\right|^{2}+(s \varphi)^{4} \lambda^{4}\left|\partial_{t} v\right|^{2}\right\} e^{-2 s \eta} d x d t \\
& \leq C \int_{Q}(s \varphi)\left(\left|\nabla \partial_{t} u_{e}\right|^{2}+\left|\partial_{t} h\right|^{2}\right) e^{-2 s \eta} d x d t+C e^{C(\lambda) s} \int_{\Sigma_{0}}\left\{\left|\partial_{t}^{2} v\right|^{2}+\left|\nabla \partial_{t} v\right|^{2}+\left|\partial_{t} v\right|^{2}\right\} d S d t
\end{aligned}
$$

Taking the time derivative to the second equation of (4.10), we get

$$
-\operatorname{div}\left(\sigma_{e} \nabla \partial_{t} u_{e}\right)=\partial_{t}^{2} v+\partial_{t} h
$$


Applying Lemma 4.2 with $p=0$ to the solution $\partial_{t} u_{e}$ of (5.4), we have

$$
\begin{aligned}
\int_{Q}\left\{(s \varphi)^{-1} \sum_{i, j=1}^{n}\left|\partial_{i} \partial_{j} \partial_{t} u_{e}\right|^{2}+(s \varphi) \lambda^{2}\left|\nabla \partial_{t} u_{e}\right|^{2}+(s \varphi)^{3} \lambda^{4}\left|\partial_{t} u_{e}\right|^{2}\right\} e^{-2 s \eta} d x d t \\
\leq C \int_{Q}\left(\left|\partial_{t}^{2} v\right|^{2}+\left|\partial_{t} h\right|^{2}\right) e^{-2 s \eta} d x d t+C e^{C(\lambda) s} \int_{\Sigma_{0}}\left\{\left|\nabla \partial_{t} u_{e}\right|^{2}+\left|\partial_{t} u_{e}\right|^{2}\right\} d S d t
\end{aligned}
$$

Using $\varphi \geq \frac{4}{T^{2}},(2 C) \times(5.3)+(5.5)$, and $\lambda$ sufficiently large, we obtain

$$
\begin{array}{r}
\int_{Q}\left\{\left|\partial_{t}^{2} v\right|^{2}+\sum_{i, j=1}^{n}\left|\partial_{i} \partial_{j} \partial_{t} v\right|^{2}+(s \varphi)^{2} \lambda^{2}\left|\nabla \partial_{t} v\right|^{2}+(s \varphi)^{4} \lambda^{4}\left|\partial_{t} v\right|^{2}+(s \varphi)^{-1} \sum_{i, j=1}^{n}\left|\partial_{i} \partial_{j} \partial_{t} u_{e}\right|^{2}\right. \\
\left.+(s \varphi) \lambda^{2}\left|\nabla \partial_{t} u_{e}\right|^{2}+(s \varphi)^{3} \lambda^{4}\left|\partial_{t} u_{e}\right|^{2}\right\} e^{-2 s \eta} d x d t \leq C \int_{Q}(s \varphi)\left|\partial_{t} h\right|^{2} e^{-2 s \eta} d x d t \\
+\tilde{C} \int_{\Sigma_{0}}\left\{\left|\partial_{t}^{2} v\right|^{2}+\left|\nabla \partial_{t} v\right|^{2}+\left|\partial_{t} v\right|^{2}+\left|\nabla \partial_{t} u_{e}\right|^{2}+\left|\partial_{t} u_{e}\right|^{2}\right\} d S d t
\end{array}
$$

Summing (4.18), (5.6) and for $\lambda$ sufficiently large, we deduce the Lemma 5.1.

Lemma 5.2. We assume that $t_{0}=T / 2$ without loss of generality by changing the scale of $t$. We get

$$
\begin{aligned}
& \int_{\Omega} e^{-2 s \eta\left(x, t_{0}\right)}\left|\mathbf{S}^{\top}\left(x, t_{0}\right) \cdot \bar{\varrho}(x)\right|^{2} d x \leq C\left(\int_{Q} s^{-1}|\bar{\varrho}(x)|^{2} e^{-2 s \eta} d x d t+\left\|v\left(\cdot, t_{0}\right)\right\|_{H^{2}(\Omega)}^{2}\right. \\
& \left.\quad+\left\|u_{e}\left(\cdot, t_{0}\right)\right\|_{H^{2}(\Omega)}^{2}+\left\|\mathbf{w}\left(\cdot, t_{0}\right)\right\|_{L^{2}(\Omega)^{k}}^{2}+\left\|\mathbf{z}\left(\cdot, t_{0}\right)\right\|_{L^{2}(\Omega)^{m}}^{2}+\|v\|_{H^{2}\left(0, T ; H^{1}\left(\Gamma_{0}\right)\right)}^{2}+\left\|u_{e}\right\|_{H^{1}\left(0, T ; H^{1}\left(\Gamma_{0}\right)\right)}^{2}\right) .
\end{aligned}
$$

Proof 5.2. We evaluate the first equation of (4.10) at a fixed time $t_{0}$, we have

$$
\left(1+\sigma_{i} \sigma_{e}^{-1}\right) \partial_{t} v\left(x, t_{0}\right)-\operatorname{div}\left(\sigma_{i} \nabla v\left(x, t_{0}\right)\right)+\left(1+\sigma_{i} \sigma_{e}^{-1}\right) h\left(x, t_{0}\right)=-\boldsymbol{A}(x) \cdot \nabla u_{e}\left(x, t_{0}\right) .
$$

Then, we integrate over $\Omega$ the square of (5.8) with the weight function $e^{-2 s \eta\left(x, t_{0}\right)}$, we obtain

$$
\begin{gathered}
\int_{\Omega} e^{-2 s \eta\left(x, t_{0}\right)}\left|\mathbf{S}^{\top}\left(x, t_{0}\right) \cdot \bar{\varrho}(x)\right|^{2} d x \leq C\left(\int_{\Omega} e^{-2 s \eta\left(x, t_{0}\right)}\left|\partial_{t} v\left(x, t_{0}\right)\right|^{2} d x\right. \\
\left.+\int_{\Omega} e^{-2 s \eta\left(x, t_{0}\right)}\left|\operatorname{div}\left(\sigma_{i} \nabla v\left(x, t_{0}\right)\right)\right|^{2} d x+\int_{\Omega} e^{-2 s \eta\left(x, t_{0}\right)}\left|\nabla u_{e}\left(x, t_{0}\right)\right|^{2} d x+\int_{\Omega} e^{-2 s \eta\left(x, t_{0}\right)}\left|R\left(x, t_{0}\right)\right|^{2} d x\right) \\
\leq C\left(\int_{\Omega} e^{-2 s \eta\left(x, t_{0}\right)}\left(\left|\partial_{t} v\left(x, t_{0}\right)\right|^{2}+\left|\nabla u_{e}\left(x, t_{0}\right)\right|^{2}+\left|R\left(x, t_{0}\right)\right|^{2}\right) d x+\left\|v\left(\cdot, t_{0}\right)\right\|_{H^{2}(\Omega)}^{2}\right)
\end{gathered}
$$

Similarly, for the second equation of (4.10) at a fixed time $t_{0}$, we get

$$
-\operatorname{div}\left(\sigma_{e} \nabla u_{e}\left(x, t_{0}\right)\right)=h\left(x, t_{0}\right)+\partial_{t} v\left(x, t_{0}\right) .
$$


Then

$$
\begin{aligned}
& \int_{\Omega} e^{-2 s \eta\left(x, t_{0}\right)}\left|\mathbf{S}^{\top}\left(x, t_{0}\right) \cdot \bar{\varrho}(x)\right|^{2} d x \leq C\left(\int_{\Omega} e^{-2 s \eta\left(x, t_{0}\right)}\left|\partial_{t} v\left(x, t_{0}\right)\right|^{2} d x\right. \\
+ & \left.\int_{\Omega} e^{-2 s \eta\left(x, t_{0}\right)}\left|\operatorname{div}\left(\sigma_{e} \nabla u_{e}\left(x, t_{0}\right)\right)\right|^{2} d x+\int_{\Omega} e^{-2 s \eta\left(x, t_{0}\right)}\left|\nabla u_{e}\left(x, t_{0}\right)\right|^{2} d x+\int_{\Omega} e^{-2 s \eta\left(x, t_{0}\right)}\left|R\left(x, t_{0}\right)\right|^{2} d x\right) .
\end{aligned}
$$

Combining (5.9) and (5.11), we have

$$
\begin{aligned}
\int_{\Omega} e^{-2 s \eta\left(x, t_{0}\right)}\left|\mathbf{S}^{\top}\left(x, t_{0}\right) \cdot \bar{\varrho}(x)\right|^{2} d x \leq C\left(\int_{\Omega} e^{-2 s \eta\left(x, t_{0}\right)}\left|\partial_{t} v\left(x, t_{0}\right)\right|^{2} d x+\left\|v\left(\cdot, t_{0}\right)\right\|_{H^{2}(\Omega)}^{2}\right. & \\
& \left.+\left\|u_{e}\left(\cdot, t_{0}\right)\right\|_{H^{2}(\Omega)}^{2}+\left\|\mathbf{w}\left(\cdot, t_{0}\right)\right\|_{L^{2}(\Omega)^{k}}^{2}+\left\|\mathbf{z}\left(\cdot, t_{0}\right)\right\|_{L^{2}(\Omega)^{m}}^{2}\right) .
\end{aligned}
$$

since

$$
\int_{\Omega} e^{-2 s \eta\left(x, t_{0}\right)}\left|R\left(x, t_{0}\right)\right|^{2} d x \leq C\left(\left\|v\left(\cdot, t_{0}\right)\right\|_{L^{2}(\Omega)}^{2}+\left\|\mathbf{w}\left(\cdot, t_{0}\right)\right\|_{L^{2}(\Omega)^{k}}^{2}+\left\|\mathbf{z}\left(\cdot, t_{0}\right)\right\|_{L^{2}(\Omega)^{m}}^{2}\right) .
$$

For the first term in the RHS of (5.12), we have

$$
\begin{array}{r}
\int_{\Omega} e^{-2 s \eta\left(x, t_{0}\right)}\left|\partial_{t} v\left(x, t_{0}\right)\right|^{2} d x=\int_{0}^{t_{0}} \int_{\Omega} \frac{d}{d t}\left(e^{-2 s \eta(x, t)}\left|\partial_{t} v\right|^{2}\right) d x d t \\
=\int_{0}^{t_{0}} \int_{\Omega}\left(-2 s \partial_{t} \eta(x, t)\left|\partial_{t} v\right|^{2}+2 p \partial_{t}^{2} v\right) e^{-2 s \eta(x, t)} d x d t \\
\leq C \int_{0}^{t_{0}} \int_{\Omega}\left(s^{2} \varphi^{2}\left|\partial_{t} v\right|^{2}+s^{-2} \varphi^{-1}\left|\partial_{t}^{2} v\right|^{2}\right) e^{-2 s \eta(x, t)} d x d t
\end{array}
$$

Then, we apply the Carleman inequality (5.1) satisfied by $\partial_{t} v$, using $\frac{4}{T^{2}} \leq \varphi \leq\left(\frac{T}{2}\right)^{2} \varphi^{2}$, we obtain for $s$ and $\lambda$ sufficiently large

$$
\begin{aligned}
& \int_{\Omega} e^{-2 s \eta\left(x, t_{0}\right)}\left|\partial_{t} v\left(x, t_{0}\right)\right|^{2} d x \leq \frac{C}{s^{2}} \int_{Q}\left(s \varphi\left|\partial_{t} h\right|^{2}+(s \varphi)^{2}|h|^{2}\right) e^{-2 s \eta} d x d t \\
& \quad+\tilde{C} \int_{\Sigma_{0}}\left\{\left|\partial_{t}^{2} v\right|^{2}+\left|\partial_{t} v\right|^{2}+\left|\nabla \partial_{t} v\right|^{2}+|\nabla v|^{2}+|v|^{2}+\left|\nabla \partial_{t} u_{e}\right|^{2}+\left|\partial_{t} u_{e}\right|^{2}+\left|\nabla u_{e}\right|^{2}+\left|u_{e}\right|^{2}\right\} d S d t
\end{aligned}
$$

Using the Lemma 5.1 given in [2], we have

$$
|h|^{2} \leq C\left(\left|\mathbf{S}^{\top} \cdot \bar{\varrho}(x)\right|^{2}+|v|^{2}+|\mathbf{w}|^{2}+|\mathbf{z}|^{2}\right),
$$

and

$$
\begin{aligned}
\left|\partial_{t} h\right|^{2} & \leq C\left(\left|\partial_{\mathbf{t}} \mathbf{S}^{\top} \cdot \bar{\varrho}(x)\right|^{2}+\left|\partial_{t} v\right|^{2}+|v|^{2}+\left|\partial_{t} \mathbf{w}\right|^{2}+|\mathbf{w}|^{2}+\left|\partial_{t} \mathbf{z}\right|^{2}+|\mathbf{z}|^{2}\right) \\
& \leq C\left(|\varrho(x)|^{2}+\left|\partial_{t} v\right|^{2}+|v|^{2}+|\mathbf{w}|^{2}+|\mathbf{z}|^{2}\right),
\end{aligned}
$$

we complete the proof.

Proof of Theorem 3.1.

In order to prove (3.5), we use the following integro-differential equation

$$
\left.v=\left\{\operatorname{div}\left(\boldsymbol{\sigma}_{\mathrm{i}} \nabla\right)\right\}^{-1}\left(\operatorname{div}\left(\boldsymbol{\sigma}_{\mathrm{i}}+\boldsymbol{\sigma}_{\mathrm{e}}\right) \nabla u_{e}\right)\right):=B u_{e},
$$


We can see that the operator $B$ is linear and uniformly continuous [11]. Replacing the new expression of $v$ in the right hand side of (5.7) and using the uniform continuity of $B$, the estimate (5.7) becomes

$$
\begin{aligned}
\int_{\Omega} e^{-2 s \eta\left(x, t_{0}\right)} \mid \mathbf{S}^{\top}\left(x, t_{0}\right) & \left.\cdot \bar{\varrho}(x)\right|^{2} d x \leq C\left(\int_{Q} s^{-1}|\bar{\varrho}(x)|^{2} e^{-2 s \eta} d x d t\right. \\
& \left.+\left\|u_{e}\left(\cdot, t_{0}\right)\right\|_{H^{2}(\Omega)}^{2}+\left\|\mathbf{w}\left(\cdot, t_{0}\right)\right\|_{L^{2}(\Omega)^{k}}^{2}+\left\|\mathbf{z}\left(\cdot, t_{0}\right)\right\|_{L^{2}(\Omega)^{m}}^{2}+\left\|u_{e}\right\|_{H^{2}\left(0, T ; H^{1}\left(\Gamma_{0}\right)\right)}^{2}\right) .
\end{aligned}
$$

Summing up the above estimate over $\ell=1, \ldots, N$, we get that

$$
\begin{aligned}
\int_{\Omega} e^{-2 s \eta\left(x, t_{0}\right)} \mid & \left.\Lambda(x) \cdot \bar{\varrho}(x)\right|^{2} d x \leq C\left(N s^{-1} \int_{\Omega} e^{-2 s \eta\left(x, t_{0}\right)}|\bar{\varrho}(x)|^{2} d x\right. \\
& \left.+\sum_{\ell=1}^{N}\left(\left\|u_{e}\right\|_{H^{2}\left(0, T ; H^{1}\left(\Gamma_{0}\right)\right)}^{2}+\left\|u_{e}\left(\cdot, t_{0}\right)\right\|_{H^{2}(\Omega)}^{2}+\left\|\mathbf{w}\left(\cdot, t_{0}\right)\right\|_{L^{2}(\Omega)^{k}}^{2}+\left\|\mathbf{z}\left(\cdot, t_{0}\right)\right\|_{L^{2}(\Omega)^{m}}^{2}\right)\right)
\end{aligned}
$$

where the $N \times N$ real matrix $\Lambda(x)=\Lambda\left(v_{\ell}^{(2)}\left(x, t_{0}\right), \mathbf{w}_{\ell}^{(2)}\left(x, t_{0}\right), \mathbf{z}_{\ell}^{(2)}\left(x, t_{0}\right)\right)$, for $x \in \Omega$. Notice that we have

$$
\|\Lambda(x) \xi\|_{\mathbb{R}^{N}} \geq \alpha_{1}(x)|\xi|_{\mathbb{R}^{N}}, \quad x \in \Omega, \quad \xi \in \mathbb{R}^{N},
$$

where $\left(\alpha_{j}(x)\right)_{1 \leq j \leq N} \subset \mathbb{R}_{+}^{N}$ denotes the increasing sequence of the singular values of $\Lambda(x)$, and $|\xi|_{\mathbb{R}^{N}}$ stands for the Euclidian norm of $\xi$. Moreover, by Sobolev embedding theorem (e.g., Thm. 5.4 in [3], Cor. 9.1, p. 46, inVol. 1 of $[35])$, we see that $H^{3}(\Omega) \subset \mathcal{C}^{1}(\bar{\Omega})$, hence $v_{\ell}^{(2)}\left(., t_{0}\right), \mathbf{w}_{\ell}^{(2)}\left(., t_{0}\right)$ and $\log \mathbf{z}_{\ell}^{(2)}\left(., t_{0}\right), \ell=1, \ldots, N$, being taken in $\mathcal{C}^{1}(\bar{\Omega})$ thanks to Proposition 2.4. Then, $\alpha_{1} \in \mathcal{C}^{1}\left(\bar{\Omega} ; \mathbb{R}_{+}\right)$from [30] [Thm 6.8 p. 122]. This combined with equation (3.4), yields $\alpha_{0}:=\inf _{x \in \Omega} \alpha_{1}(x)>0$. As a Consequence and by (5.20), we have

$$
\int_{\Omega} e^{-2 s \eta\left(x, t_{0}\right)}|\Lambda(x) \bar{\varrho}(x)|^{2} d x \geq \alpha_{0}^{2} \int_{\Omega} e^{-2 s \eta\left(x, t_{0}\right)}|\bar{\varrho}(x)|^{2} d x,
$$

and Theorem 3.1 follows directly from this and (5.19) by choosing $s$ so large that $C N s^{-1}<\alpha_{0}^{2}$.

\section{Discussion and COnClusions}

Personalizing a numerical model in cardiac electrophysiology simulation allows to assimilate the electrophysiology conditions of a patient, including anatomical information but also functional conditions. These functional conditions are generally observed in the electrical signals measured either invasively in the heart domain or non-invasively on the body surface. The underlying substrate behind the electrical changes could be related to macroscopic parameters like the conductivity distribution of the tissue, but also and most importantly to the microscopic parameters at the cell and sub-cell levels. In particular, the conductances of the ion channels play an important role in the modulation of the transmembrane potential and consequently the ECGs. In this paper, we addressed the problem of identifying ion channels conductances from a set of extracellular potentials measurements on the boundary of the heart. Our result says that it is possibly to uniquely identify the conductances parameters if the mathematical model governing the electrical activity satisfies some hypothesis. Our approach is based on a Carleman inequality for the bidomain reaction diffusion model coupled to a general form of ordinary differential equation system. This result is a step further in the parameters identification problem compared to the existing results $[2,4,10,33,51]$. There are two novelties in our result compared to the results in those paper. First the cited papers deal with simpler models either by simplifying the ionic model or by simplifying the anisotropy of the bidomain model to reach the condition of the monodomain model approximation. In our case, we only remove the anisotropy but we keep ratio between intra and extracellular conductivities space dependent and we consider physiologically detailed ionic model. Second, we consider that the observations are 
given on a part of the accessible boundary of the domain which is more realistic compared to observations on a sub-domain of the heart.

Acknowledgements. This work has been supported by EPICARD cooperative research program, funded by INRIA international laboratory LIRIMA. The LAMSIN researcher's work is supported on a regular basis by the Tunisian Ministry of Higher Education, Scientific Research and Technology. This study received also financial support from the French Government as part of the 'Investments of the future' program managed by the National Research Agency (ANR), Grant reference ANR-10-IAHU-04.

\section{Appendix}

Proof of Proposition 2.4.

First, we propose a parabolic-elliptic formulation of (2.1) given as follow:

$$
\left\{\begin{array}{lc}
\partial_{t} v-\operatorname{div}\left(\boldsymbol{\sigma}_{i} \nabla v\right)+I_{i o n}(\bar{\varrho}, v, \mathbf{w}, \mathbf{z})=\operatorname{div}\left(\boldsymbol{\sigma}_{i} \nabla u_{e}\right)+I_{i}^{s} & \text { in } Q, \\
-\operatorname{div}\left(\boldsymbol{\sigma}_{i} \nabla v+\left(\boldsymbol{\sigma}_{i}+\boldsymbol{\sigma}_{e}\right) \nabla u_{e}\right)=I_{i}^{s}+I_{e}^{s} & \text { in } Q, \\
\partial_{t} \mathbf{w}=\boldsymbol{F}(v, \mathbf{w}) & \text { in } Q, \\
\partial_{t} \mathbf{z}=\boldsymbol{G}(\bar{\varrho}, v, \mathbf{w}, \mathbf{z}) & \text { in } Q, \\
\boldsymbol{\sigma}_{i} \nabla v \cdot \nu+\boldsymbol{\sigma}_{i} \nabla u_{e} \cdot \nu=0 & \text { on } \Sigma, \\
\boldsymbol{\sigma}_{i} \nabla v \cdot \nu+\left(\boldsymbol{\sigma}_{i}+\boldsymbol{\sigma}_{e}\right) \nabla u_{e} \cdot \nu=0 & \text { on } \Sigma, \\
v(x, 0)=v_{0}(x), u_{e}(x, 0)=u_{e, 0}(x), \mathbf{w}(x, 0)=\mathbf{w}_{0}(x), \mathbf{z}(x, 0)=\mathbf{z}_{0}(x) & \text { in } \Omega .
\end{array}\right.
$$

Taking the time derivative of equations system (7.1), we obtain

$$
\begin{cases}\partial_{t t} v-\operatorname{div}\left(\boldsymbol{\sigma}_{i} \nabla \partial_{t} v\right)+\partial_{t} I_{i o n}=\operatorname{div}\left(\boldsymbol{\sigma}_{i} \nabla \partial_{t} u_{e}\right)+\partial_{t} I_{i}^{s} & \text { in } Q, \\ -\operatorname{div}\left(\boldsymbol{\sigma}_{i} \nabla \partial_{t} v+\left(\boldsymbol{\sigma}_{i}+\boldsymbol{\sigma}_{e}\right) \nabla \partial_{t} u_{e}\right)=\partial_{t} I_{i}^{s}+\partial_{t} I_{e}^{s} & \text { in } Q, \\ \partial_{t t} \mathbf{w}=\partial_{t} \boldsymbol{F}(v, \mathbf{w}) & \text { in } Q, \\ \partial_{t t} \mathbf{z}=\partial_{t} \boldsymbol{G}(\bar{\varrho}, v, \mathbf{w}, \mathbf{z}) & \text { in } Q, \\ \boldsymbol{\sigma}_{i} \nabla \partial_{t} v \cdot \nu+\boldsymbol{\sigma}_{i} \nabla \partial_{t} u_{e} \cdot \nu=0 & \text { on } \Sigma, \\ \boldsymbol{\sigma}_{i} \nabla \partial_{t} v \cdot \nu+\left(\boldsymbol{\sigma}_{i}+\boldsymbol{\sigma}_{e}\right) \nabla \partial_{t} u_{e} \cdot \nu=0 & \text { on } \Sigma,\end{cases}
$$

with initial conditions

$$
\begin{array}{rlrl}
\partial_{t} v(t=0) & =\operatorname{div}\left(\boldsymbol{\sigma}_{i} \nabla v_{0}\right)+\operatorname{div}\left(\boldsymbol{\sigma}_{i} \nabla u_{e}(t=0)\right)+I_{i}^{s}(t=0)-I_{\text {ion }}(t=0) & & \text { in } \Omega, \\
\partial_{t} u_{e}(t=0) & =\partial_{t} u_{e, 0} & & \text { in } \Omega, \\
\partial_{t} \mathbf{w}(t=0) & =\boldsymbol{F}\left(v_{0}, w_{0}\right) & \text { in } \Omega, \\
\partial_{t} \mathbf{z}(t=0) & =\boldsymbol{G}\left(\bar{\varrho}, v_{0}, \mathbf{w}_{0}, \mathbf{z}_{0}\right) & \text { in } \Omega .
\end{array}
$$

Using the hypothesis $v_{0} \in H^{2}(\Omega), u_{e}(t=0) \in H^{2}(\Omega), \mathbf{w}_{0} \in L^{2}(\Omega)^{k}$ and $\mathbf{z}_{0} \in L^{2}(\Omega)^{m}$, we deduce that

$$
\operatorname{div}\left(\boldsymbol{\sigma}_{i} \nabla v_{0}\right) \in L^{2}(\Omega), \quad \operatorname{div}\left(\boldsymbol{\sigma}_{i} \nabla u_{e}(t=0)\right) \in L^{2}(\Omega), \quad I_{\text {ion }}(t=0) \in L^{2}(\Omega),
$$


and then $\partial_{t} v(t=0) \in L^{2}(\Omega)$ since $I_{i, e}^{s}$ satisfy the hypothesis (2.14). Also we deduce that

$$
\partial_{t} \mathbf{w}(t=0) \in L^{2}(\Omega)^{k}, \quad \text { and } \quad \partial_{t} \mathbf{z}(t=0) \in L^{2}(\Omega)^{m} .
$$

We integrate over $\Omega$ the sum of the first equation of (7.2) multiplied by $\partial_{t} v$, the $2^{\text {nd }}, 3^{\text {rd }}$ and the $4^{\text {th }}$ one multiplied by $\partial_{t} u_{e}, \partial_{t} \mathbf{w}$ and $\partial_{t} \mathbf{z}$, respectively, we obtain

$$
\begin{array}{r}
\frac{1}{2} \frac{d}{d t}\left(\left\|\partial_{t} v\right\|_{L^{2}(\Omega)}^{2}+\left\|\partial_{t} \mathbf{w}\right\|_{L^{2}(\Omega)^{k}}^{2}+\left\|\partial_{t} \mathbf{z}\right\|_{L^{2}(\Omega)^{m}}^{2}\right)+<\boldsymbol{\sigma}_{i} \nabla \partial_{t}\left(v+u_{e}\right), \nabla \partial_{t}\left(v+u_{e}\right)>+<\boldsymbol{\sigma}_{e} \nabla \partial_{t} u_{e}, \nabla \partial_{t} u_{e}>= \\
\int_{\Omega} \partial_{t} v \partial_{t} I_{i}^{s} d x+\int_{\Omega} \partial_{t} u_{e}\left(\partial_{t} I_{i}^{s}+\partial_{t} I_{e}^{s}\right) d x+\int_{\Omega} \partial_{t} v \partial_{t} I_{i o n} d x+\int_{\Omega} \partial_{t} \mathbf{w} \cdot \partial_{t} \boldsymbol{F} d x+\int_{\Omega} \partial_{t} \mathbf{z} . \partial_{t} \boldsymbol{G} d x .
\end{array}
$$

where $\langle\boldsymbol{\sigma} \nabla \vartheta, \nabla \vartheta\rangle=\int_{\Omega}(\sigma \nabla \vartheta)^{T} \nabla \vartheta d x$. From Assumptions 2.1, we consider

$$
<\boldsymbol{\sigma}_{i} \nabla \partial_{t}\left(v+u_{e}\right), \nabla \partial_{t}\left(v+u_{e}\right)>\geq \underline{\sigma_{i}}\left\|\nabla \partial_{t} v+\nabla \partial_{t} u_{e}\right\|_{L^{2}(\Omega)}^{2},
$$

and

$$
<\boldsymbol{\sigma}_{e} \nabla \partial_{t} u_{e}, \nabla \partial_{t} u_{e}>\geq \underline{\sigma_{e}}\left\|\nabla \partial_{t} u_{e}\right\|_{L^{2}(\Omega)}^{2} .
$$

Using Cauchy-Schwarz inequality, we have

$$
\begin{gathered}
\int_{\Omega} \partial_{t} v \partial_{t} I_{i}^{s} d x \leq \frac{1}{2}\left(\left\|\partial_{t} v\right\|_{L^{2}(\Omega)}^{2}+\left\|\partial_{t} I_{i}^{s}\right\|_{L^{2}(\Omega)}^{2}\right) \\
\int_{\Omega} \partial_{t} u_{e} \partial_{t}\left(I_{i}^{s}+I_{e}^{s}\right) d x \leq \epsilon \partial_{t} u_{e} \|_{L^{2}(\Omega)}^{2}+C_{\epsilon}\left(\left\|\partial_{t} I_{i}^{s}\right\|_{L^{2}(\Omega)}^{2}+\left\|\partial_{t} I_{e}^{s}\right\|_{L^{2}(\Omega)}^{2}\right),
\end{gathered}
$$

Computing the time derivative of the ionic curren $I_{i o n}$, we get

$$
\begin{aligned}
\partial_{t} I_{\text {ion }}= & \sum_{i=1}^{N} \bar{\varrho}_{i} \partial_{t} v y_{i}^{\prime}(v) \prod_{j=1}^{k} w_{j}^{p_{j, i}}\left(v-E_{i}(\mathbf{z})\right) \\
& +\sum_{i=1}^{N} \bar{\varrho}_{i} y_{i}(v)\left(\sum_{l=1}^{k} p_{l, i} w_{l}^{p_{l, i}-1} \partial_{t} w_{l} \prod_{j \neq l} w_{j}^{p_{j, i}}\right)\left(v-E_{i}(\mathbf{z})\right) \\
& +\sum_{i=1}^{N} \bar{\varrho}_{i} y_{i}(v) \partial_{t} v \prod_{j=1}^{k} w_{j}^{p_{j, i}}+\sum_{i=1}^{N} \bar{\varrho}_{i} \frac{\partial_{t} z_{i}}{z_{i}} y_{i}(v) \prod_{j=1}^{k} w_{j}^{p_{j, i}} .
\end{aligned}
$$

Thanks to Lemma 2.3, we have $v \in L^{\infty}(Q), \log \mathbf{z} \in L^{\infty}(Q)^{m}, \mathbf{w} \in[0,1]^{k}$ and $y_{i}$ is $\mathcal{C}^{\infty}$. Applying CauchySchwarz inequality, we deduce from (7.9) and (7.11) that

$$
\int_{\Omega} \partial_{t} v \partial_{t} I_{i}^{s} d x+\int_{\Omega} \partial_{t} v \partial_{t} I_{i o n} d x \leq C\left(\left\|\partial_{t} v\right\|_{L^{2}(\Omega)}^{2}+\left\|\partial_{t} \mathbf{w}\right\|_{L^{2}(\Omega)^{k}}^{2}+\left\|\partial_{t^{2}}\right\|_{L^{2}(\Omega)^{m}}^{2}+\left\|\partial_{t} I_{i}^{s}\right\|_{L^{2}(\Omega)}^{2}\right) .
$$

We recall that the function $F_{j}$ given by $(2.5)$ is $\mathcal{C}^{2}\left(\mathbb{R}^{2}\right)$ for $j=1, \ldots, k$, and then we can write

$$
\partial_{t} F_{j}\left(v, w_{j}\right)=\partial_{t} v \partial_{1} F_{j}+\partial_{t} w_{j} \partial_{2} F_{j}
$$

where $\partial_{l}$ is the partial derivative with respect the $l^{\text {th }}$ variable, $l=1,2$. Since $\alpha_{j}, \beta_{j}$ are $\mathcal{C}^{\infty}(\mathbb{R}), v \in L^{\infty}(Q)$ and $w_{j} \in[0,1], j=1, \ldots, k$, we deduce that

$$
\partial_{1} F_{j}\left(v, w_{j}\right)=\alpha^{\prime}(v)\left(1-w_{j}\right)-\beta^{\prime}(v) w_{j}, \quad \text { and } \quad \partial_{2} F_{j}\left(v, w_{j}\right)=-\alpha(v)-\beta(v), \quad j=1, \ldots, k,
$$


are bounded. Then, by Cauchy-Schwarz inequality and Young inequality, we have

$$
\int_{\Omega} \partial_{t} \mathbf{w} \cdot \partial_{t} \boldsymbol{F} d x \leq C\left(\left\|\partial_{t} v\right\|_{L^{2}(\Omega)}^{2}+\left\|\partial_{t} \mathbf{w}\right\|_{L^{2}(\Omega)^{k}}^{2}\right)
$$

Similarly for the variable $\mathbf{z}$, for $i=1, \ldots, m$, we can write

$$
\begin{aligned}
\partial_{t} G_{i}(\bar{\varrho}, v, \mathbf{w}, \mathbf{z})= & -\left(\partial_{t} v \partial_{2} J_{i}+\sum_{j=1}^{k}\left(\partial_{t} w_{j} \partial_{j+2} J_{i}\right)+\partial_{t} \log z_{i} \partial_{k+3} J_{i}\right) \\
& +\partial_{t} v \partial_{2} H_{i}+\sum_{j=1}^{k} \partial_{t} w_{j} \partial_{j+2} H_{i}+\sum_{j=1}^{m} \partial_{t} \log z_{j} \partial_{k+2+j} H_{i} .
\end{aligned}
$$

By hypothesis $(2.8)$ and $(2.9)$, we deduce that $\partial_{l} J_{i}, l=1, \ldots, k+3$, and $\partial_{l} H_{i}, l=1, \ldots, m+k+2$, are bounded since $v \in L^{\infty}(Q), \mathbf{w} \in[0,1]^{k}, \mathbf{z} \in L^{\infty}(Q)^{m}$. Then, we have

$$
\int_{\Omega} \partial_{t} \mathbf{z} \cdot \partial_{t} \boldsymbol{G} d x \leq C\left(\left\|\partial_{t} v\right\|_{L^{2}(\Omega)}^{2}+\left\|\partial_{t} \mathbf{w}\right\|_{L^{2}(\Omega)^{k}}^{2}+\left\|\partial_{t} \mathbf{z}\right\|_{L^{2}(\Omega)^{m}}^{2}\right) .
$$

Thus, substituting (7.7), (7.8), (7.10), (7.12), (7.15) and (7.17) in (7.6), and applying Poincaré inequality by choosing $\epsilon=\frac{1}{2 C_{p}} \underline{\sigma_{e}}$, with $C_{p}$ is the Poincaré constant depending on $\Omega$, we obtain

$$
\begin{aligned}
& \frac{d}{d t}\left(\left\|\partial_{t} v\right\|_{L^{2}(\Omega)}^{2}+\left\|\partial_{t} \mathbf{w}\right\|_{L^{2}(\Omega)^{k}}^{2}+\left\|\partial_{t} \mathbf{z}\right\|_{L^{2}(\Omega)^{m}}^{2}\right)+\underline{\sigma_{i}}\left\|\nabla \partial_{t} v+\nabla \partial_{t} u_{e}\right\|_{L^{2}(\Omega)}^{2}+\frac{1}{2} \underline{\sigma_{e}}\left\|\nabla \partial_{t} u_{e}\right\|_{L^{2}(\Omega)}^{2} \\
& \leq C\left(\left\|\partial_{t} v\right\|_{L^{2}(\Omega)}^{2}+\left\|\partial_{t} \mathbf{w}\right\|_{L^{2}(\Omega)^{k}}^{2}+\left\|\partial_{t} \mathbf{z}\right\|_{L^{2}(\Omega)^{m}}^{2}+\left\|\partial_{t} I_{i}^{s}\right\|_{L^{2}(\Omega)}^{2}+\left\|\partial_{t} I_{e}^{s}\right\|_{L^{2}(\Omega)}^{2}\right) .
\end{aligned}
$$

Applying Gronwall Lemma, we deduce

$$
\left\|\partial_{t} v\right\|_{L^{2}(\Omega)}^{2}+\left\|\partial_{t} \mathbf{w}\right\|_{L^{2}(\Omega)^{k}}^{2}+\left\|\partial_{t} \mathbf{z}\right\|_{L^{2}(\Omega)^{m}}^{2}+\underline{\sigma_{i}}\left\|\nabla \partial_{t} v+\nabla \partial_{t} u_{e}\right\|_{L^{2}(\Omega)}^{2}+\frac{1}{2} \underline{\sigma_{e}}\left\|\nabla \partial_{t} u_{e}\right\|_{L^{2}(\Omega)}^{2} \leq C_{0}, \quad \forall t \in[0, T]
$$

where

$$
C_{0}=C\left(\left\|v_{0}\right\|_{H^{2}(\Omega)},\left\|\mathbf{w}_{0}\right\|_{L^{2}(\Omega)^{k}},\left\|\mathbf{z}_{0}\right\|_{L^{2}(\Omega)^{m}},\left\|I_{i, e}^{s}\right\|_{H^{1}\left(0, T ; L^{2}(\Omega)\right)}\right)>0 .
$$

Thus,

$$
v, u_{e} \in W^{1, \infty}\left(0, T ; H^{1}(\Omega)\right), \quad \mathbf{w} \in W^{1, \infty}\left(0, T ; L^{2}(\Omega)\right)^{k}, \quad \text { and } \quad \mathbf{z} \in W^{1, \infty}\left(0, T ; L^{2}(\Omega)\right)^{m} .
$$

Now, for $t \in(0, T)$, we intergrate $(7.18)$ over $(0, t)$, we obtain

$$
\begin{aligned}
\left\|\partial_{t} v\right\|_{L^{2}(\Omega)}^{2}+\left\|\partial_{t} \mathbf{w}\right\|_{L^{2}(\Omega)^{k}}^{2}+\left\|\partial_{t} \mathbf{z}\right\|_{L^{2}(\Omega)^{m}}^{2} & +\underline{\sigma_{i}} \int_{0}^{t}\left\|\nabla \partial_{t} v+\nabla \partial_{t} u_{e}\right\|_{L^{2}(\Omega)}^{2} d \tau \\
& +\frac{1}{2} \underline{\sigma_{e}} \int_{0}^{t}\left\|\nabla \partial_{t} u_{e}\right\|_{L^{2}(\Omega)}^{2} d \tau \leq 2 C_{0}
\end{aligned}
$$

and then, we conclude

$$
v, u_{e} \in H^{1}\left(0, T ; H^{1}(\Omega)\right) .
$$

On the other hand, from the system (2.10), we have

$$
\begin{aligned}
\operatorname{div}\left(\boldsymbol{\sigma}_{e} \nabla u_{e}\right) & =-\partial_{t} v-I_{i o n}-I_{e}^{s} \in L^{2}\left(0, T ; L^{2}(\Omega)\right), \\
& \Rightarrow u_{e} \in L^{2}\left(0, T ; H^{2}(\Omega)\right)
\end{aligned}
$$


and

$$
\begin{gathered}
\operatorname{div}\left(\boldsymbol{\sigma}_{i} \nabla v\right)=\partial_{t} v+I_{i o n}-\operatorname{div}\left(\boldsymbol{\sigma}_{i} \nabla u_{e}\right)-I_{i}^{s} \in L^{2}\left(0, T ; L^{2}(\Omega)\right), \\
\Rightarrow v \in L^{2}\left(0, T ; H^{2}(\Omega)\right) .
\end{gathered}
$$

Let us now prove the regularities (2.21). Deriving equations (2.5) and (2.7) over the space variable $x$, we obtain

and

$$
\partial_{t}\left(\partial_{x} w_{j}\right)=\partial_{x} v \partial_{1} F_{j}+\partial_{x} w_{j} \partial_{2} F_{j}, \quad j=1, \ldots, k,
$$

$$
\partial_{t}\left(\partial_{x} z_{i}\right)=\sum_{j=1}^{N} \partial_{x} \varrho_{j} \partial_{j} G_{i}+\partial_{x} v \partial_{N+1} G_{i}+\sum_{j=1}^{k} \partial_{x} w_{j} \partial_{j+N+1} G_{i}+\sum_{j=1}^{m} \partial_{x} z_{j} \partial_{k+N+1+j} G_{i}, \quad i=1, \ldots, m .
$$

Without loss of generality, $\partial_{x}$ is the space derivative over one direction (here could be the first, the second or the third dimension of the space $\mathbb{R}^{3}$ ).

According to Lemma 2.3 and the hypothesis (2.7)-(2.9), there exist a constant $C$ depending on $T$, such that

$$
\left\|\partial_{1} F_{j}\right\|_{L^{2}(Q)}^{2}+\left\|\partial_{2} F_{j}\right\|_{L^{2}(Q)}^{2} \leq C, \quad j=1, \ldots, k,
$$

and

$\sum_{j=1}^{N}\left\|\partial_{x} \varrho_{j} \partial_{j} G_{i}\right\|_{L^{2}(Q)}^{2}+\left\|\partial_{N+1} G_{i}\right\|_{L^{2}(Q)}^{2}+\sum_{j=1}^{k}\left\|\partial_{j+N+1} G_{i}\right\|_{L^{2}(Q)}^{2}+\sum_{j=1}^{m}\left\|\partial_{k+N+1+j} G_{i}\right\|_{L^{2}(Q)}^{2} \leq C, \quad i=1, \ldots, m$.

Multiplying the equation $(7.26)$ by $\left(\partial_{x} w_{j}\right), j=1, \ldots, k$, the equation $(7.27)$ by $\left(\partial_{x} z_{i}\right), i=1, \ldots, m$, and integrating the sum over $\Omega$, and applying the Young inequality of the right hand side, we obtain

$$
\frac{d}{d t}\left(\left\|\partial_{x} \mathbf{w}\right\|_{L^{2}(\Omega)^{k}}^{2}+\left\|\partial_{x} \mathbf{z}\right\|_{L^{2}(\Omega)^{m}}^{2}\right) \leq C\left(\left\|\partial_{x} v\right\|_{L^{2}(\Omega)}^{2}+\left\|\partial_{x} \mathbf{w}\right\|_{L^{2}(\Omega)^{k}}^{2}+\left\|\partial_{x} \mathbf{z}\right\|_{L^{2}(\Omega)^{m}}^{2}\right) .
$$

Thanks to the regularity (7.23), we have $\left\|\partial_{x} v\right\|_{L^{2}(\Omega)} \in C^{0}[0, T]$. Applying Gronwall lemma and using hypothesis (2.20), we obtain

$$
\left\|\partial_{x} \mathbf{w}\right\|_{L^{2}(\Omega)^{k}}^{2}+\left\|\partial_{x} \mathbf{z}\right\|_{L^{2}(\Omega)^{m}}^{2} \leq C\left(\left\|\partial_{x} \mathbf{w}_{0}\right\|_{L^{2}(\Omega)^{k}},\left\|\partial_{x} \mathbf{z}_{0}\right\|_{L^{2}(\Omega)^{m}}\right), \quad \forall t \in[0, T] .
$$

Thus,

$$
\mathbf{w} \in L^{\infty}\left(0, T ; H^{1}(\Omega)\right)^{k}, \quad \text { and } \quad \mathbf{z} \in L^{\infty}\left(0, T ; H^{1}(\Omega)\right)^{m} .
$$

On the other hand, from (7.26) and (7.27), we deduce

$$
\mathbf{w} \in W^{1, \infty}\left(0, T ; H^{1}(\Omega)\right)^{k}, \quad \text { and } \quad \mathbf{z} \in W^{1, \infty}\left(0, T ; H^{1}(\Omega)\right)^{m} .
$$

- Let us now consider the initial conditions:

- $\partial_{t t} v(0)=\operatorname{div}\left(\boldsymbol{\sigma}_{i} \nabla \partial_{t} v(0)\right)+\operatorname{div}\left(\boldsymbol{\sigma}_{i} \nabla \partial_{t} u_{e}(0)\right)+\partial_{t} I_{i}^{s}(0)-\partial_{t} I_{i o n}(0)$,

- $\partial_{t t} \mathbf{w}(0)=\partial_{t} \boldsymbol{F}(v(0), \mathbf{w}(0))$,

- $\partial_{t t} \mathbf{z}(0)=\partial_{t} \boldsymbol{G}(\bar{\varrho}, v(0), \mathbf{w}(0), \mathbf{z}(0))$.

We start by showing that $\partial_{t} v(t=0) \in H^{2}(\Omega)$. Using hypothesis $(2.14), v_{0} \in H^{4}(\Omega)$, and $u_{e}(t=0) \in H^{4}(\Omega)$, we have $\operatorname{div}\left(\sigma_{i} \nabla v_{0}\right) \in H^{2}(\Omega)$, and $\operatorname{div}\left(\sigma_{i} \nabla u_{e}(t=0)\right) \in H^{2}(\Omega)$. We also have $I_{i}^{s}(t=0) \in H^{2}(\Omega)$. It's obvious 
that

$$
I_{i o n}(t=0)=\sum_{i=1}^{N} \bar{\varrho}_{i} y_{i}\left(v_{0}\right) \prod_{j=1}^{k} w_{0, j}^{p_{j, i}}\left(v_{0}-E_{i}\left(\mathbf{z}_{0}\right)\right) \in L^{2}(\Omega) .
$$

Since $\bar{\varrho}$ is bounded in $H^{3}(\Omega)^{N}$, there exists a constant $C>0$ such that

$$
\begin{aligned}
\left|\partial_{x} I_{i o n}(t=0)\right|^{2} & \leq C\left(\left|\partial_{x} v_{0}\right|^{2}+\left|\partial_{x} \mathbf{w}_{0}\right|^{2}+\left|\partial_{x} \mathbf{z}_{0}\right|^{2}\right) \\
\left|\partial_{x y} I_{i o n}(t=0)\right|^{2} & \leq C\left(\left|\partial_{x} v_{0}\right|^{2}+\left|\partial_{x} \mathbf{w}_{0}\right|^{2}+\left|\partial_{x} \mathbf{z}_{0}\right|^{2}+\left|\partial_{y} v_{0}\right|^{2}+\left|\partial_{y} \mathbf{w}_{0}\right|^{2}+\left|\partial_{y} \mathbf{z}_{0}\right|^{2}\right. \\
& \left.+\left|\partial_{x y} v_{0}\right|^{2}+\left|\partial_{x y} \mathbf{w}_{0}\right|^{2}+\left|\partial_{x y} \mathbf{z}_{0}\right|^{2}\right)
\end{aligned}
$$

So, from hypothesis $v_{0} \in H^{4}(\Omega), \mathbf{w}_{0} \in H^{2}(\Omega)^{k}, \mathbf{z}_{0} \in H^{2}(\Omega)^{m}$, we deduce that $\partial_{x} I_{\text {ion }}(t=0)$ and $\partial_{x y} I_{i o n}(t=0)$ belong to $L^{2}(\Omega)$. Thus $I_{\text {ion }}(t=0) \in H^{2}(\Omega)$, and then $\partial_{t} v(t=0) \in H^{2}(\Omega)$. From $(7.34)$, we get $\partial_{t t} v(t=0) \in$ $L^{2}(\Omega), \partial_{t t} \mathbf{w}(t=0) \in L^{2}(\Omega)^{k}$, and $\partial_{t t} \mathbf{z}(t=0) \in L^{2}(\Omega)^{m}$.

Now, we take the second derivative with respect to time of the system (7.1), we multiply the first (respectively the $2^{n d}, 3^{r d}$ and the $4^{t h}$ ) equation by $\partial_{t t} v$ (respectively by $\partial_{t t} u_{e}, \partial_{t t} \mathbf{w}, \partial_{t t} \mathbf{z}$ ), we obtain

- $\partial_{t t t} v \partial_{t t} v-\partial_{t t} v \operatorname{div}\left(\boldsymbol{\sigma}_{i} \nabla \partial_{t t} v\right)=\partial_{t t} v \operatorname{div}\left(\boldsymbol{\sigma}_{i} \nabla \partial_{t t} u_{e}\right)+\partial_{t t} v \partial_{t t} I_{i}^{s}-\partial_{t t} v \partial_{t t} I_{i o n}$,

- $-\partial_{t t} u_{e} \operatorname{div}\left(\boldsymbol{\sigma}_{i} \nabla \partial_{t t} v\right)-\partial_{t t} u_{e} \operatorname{div}\left(\left(\boldsymbol{\sigma}_{i}+\boldsymbol{\sigma}_{e}\right) \nabla \partial_{t t} u_{e}\right)=\partial_{t t} u_{e}\left(\partial_{t t} I_{i}^{s}+\partial_{t t} I_{e}^{s}\right)$,

- $\partial_{t t t} w_{j} \partial_{t t} w_{j}=\partial_{t t} F_{j}\left(v, w_{j}\right) \partial_{t t} w_{j}, \quad j=1, \cdots, k$,

- $\partial_{t t t} z_{i} \partial_{t t} z_{i}=\partial_{t t} G_{i}(\bar{\varrho}, v, \mathbf{w}, \mathbf{z}) \partial_{t t} z_{i}, \quad i=1, \cdots, m$.

Deriving (7.11) with respect to time, integrating over $\Omega$ the sum of the first and second equation of (7.37), using Cauchy Schwarz inequality, we get

$$
\begin{aligned}
& \frac{1}{2} \frac{d}{d t}\left\|\partial_{t t} v\right\|_{L^{2}(\Omega)}^{2}+\frac{1}{2}<\boldsymbol{\sigma}_{i} \nabla \partial_{t t}\left(v+u_{e}\right), \nabla \partial_{t t}\left(v+u_{e}\right)>+\frac{1}{2}<\boldsymbol{\sigma}_{e} \nabla \partial_{t t} u_{e}, \nabla \partial_{t t} u_{e}> \\
& \leq C_{1}\left(\left\|\partial_{t t} v\right\|_{L^{2}(\Omega)}^{2}+\left\|\partial_{t t} u_{e}\right\|_{L^{2}(\Omega)}^{2}\right)+C_{2}\left\|\partial_{t t} \mathbf{w}\right\|_{L^{2}(\Omega)^{k}}^{2}+C_{3}\left\|\partial_{t t} \mathbf{z}\right\|_{L^{2}(\Omega)^{m}}^{2} \\
& +C_{4}\left(\left\|\partial_{t} v\right\|_{L^{2}(\Omega)}^{2}+\left\|\partial_{t} \mathbf{w}\right\|_{L^{2}(\Omega)^{k}}^{2}+\left\|\partial_{t} \mathbf{z}\right\|_{L^{2}(\Omega)^{m}}^{2}+\left\|\partial_{t t} I_{i}^{s}\right\|_{L^{2}(\Omega)}^{2}+\left\|\partial_{t t} I_{e}^{s}\right\|_{L^{2}(\Omega)}^{2}\right) .
\end{aligned}
$$

Deriving in time (7.13) and (7.16), integrating over $\Omega$ the $3^{\text {rd }}$ and the $4^{\text {th }}$ equation of (7.37), using Cauchy Schwarz inequality, for $j=1, \ldots, k$ and $i=1, \ldots, m$, we get

- $\frac{1}{2} \frac{d}{d t}\left\|\partial_{t t} w_{j}\right\|_{L^{2}(\Omega)}^{2}+C_{\alpha, \beta}\left\|\partial_{t t} w_{j}\right\|_{L^{2}(\Omega)}^{2} \leq C_{5}\left\|\partial_{t t} v\right\|_{L^{2}(\Omega)}^{2}+C_{6}\left\|\partial_{t t} w_{j}\right\|_{L^{2}(\Omega)}^{2}$ $+C_{7}\left(\left\|\partial_{t} v\right\|_{L^{2}(\Omega)}^{2}+\left\|\partial_{t} w_{j}\right\|_{L^{2}(\Omega)}^{2}\right)$,

- $\frac{1}{2} \frac{d}{d t}\left\|\partial_{t t} z_{i}\right\|_{L^{2}(\Omega)}^{2}+\underline{C}\left\|\partial_{t t} z_{i}\right\|_{L^{2}(\Omega)}^{2} \leq C_{8}\left\|\partial_{t t} v\right\|_{L^{2}(\Omega)}^{2}+C_{9}\left\|\partial_{t t} \mathbf{w}\right\|_{L^{2}(\Omega)^{k}}^{2}+C_{10}\left\|\partial_{t t} z_{i}\right\|_{L^{2}(\Omega)}^{2}$ $+C_{11}\left(\left\|\partial_{t} v\right\|_{L^{2}(\Omega)}^{2}+\left\|\partial_{t} \mathbf{w}\right\|_{L^{2}(\Omega)^{k}}^{2}+\left\|\partial_{t} z_{i}\right\|_{L^{2}(\Omega)}^{2}\right)$, 
where $C_{1}, C_{2}, \ldots, C_{11}, C_{\alpha, \beta}, \underline{C}$ positive constants such that $\alpha_{j}(v)+\beta_{j}(v) \geq C_{\alpha, \beta}$ and $\frac{1}{z_{i}} \partial_{k+3} J_{i} \geq \underline{C}$. Summing all equations in (7.38) and (7.39), we get

$$
\begin{aligned}
& \frac{1}{2} \frac{d}{d t}\left(\left\|\partial_{t t} v\right\|_{L^{2}(\Omega)}^{2}+\left\|\partial_{t t} \mathbf{w}\right\|_{L^{2}(\Omega)^{k}}^{2}+\left\|\partial_{t t} \mathbf{z}\right\|_{L^{2}(\Omega)^{m}}^{2}\right)+\frac{1}{2} \underline{\sigma_{i}}\left\|\nabla \partial_{t t} v+\nabla \partial_{t t} u_{e}\right\|_{L^{2}(\Omega)}^{2}+\frac{1}{2} \underline{\sigma_{e}}\left\|\nabla \partial_{t t} u_{e}\right\|_{L^{2}(\Omega)}^{2} \\
& +C_{\alpha, \beta}\left\|\partial_{t t} \mathbf{w}\right\|_{L^{2}(\Omega)^{k}}^{2}+\underline{C}\left\|\partial_{t t} \mathbf{z}\right\|_{L^{2}(\Omega)^{m}}^{2} \leq C_{12}\left(\left\|\partial_{t t} v\right\|_{L^{2}(\Omega)}^{2}+\left\|\partial_{t t} u_{e}\right\|_{L^{2}(\Omega)}^{2}\right)+C_{13}\left\|\partial_{t t} \mathbf{w}\right\|_{L^{2}(\Omega)^{k}}^{2} \\
& +C_{14}\left\|\partial_{t t} \mathbf{z}\right\|_{L^{2}(\Omega)^{m}}^{2}+C_{15}\left(\left\|\partial_{t} v\right\|_{L^{2}(\Omega)}^{2}+\left\|\partial_{t} \mathbf{w}\right\|_{L^{2}(\Omega)^{k}}^{2}+\left\|\partial_{t} \mathbf{z}\right\|_{L^{2}(\Omega)^{m}}^{2}+\left\|\partial_{t t} I_{i}^{s}\right\|_{L^{2}(\Omega)}^{2}+\left\|\partial_{t t} I_{e}^{s}\right\|_{L^{2}(\Omega)}^{2}\right) .
\end{aligned}
$$

Applying Poincaré inequality by choosing $C_{12}=\frac{1}{4 C_{p}} \underline{\sigma_{i}}$, with $C_{p}$ is the Poincaré constant depending on $\Omega$, $C_{13}=\frac{1}{2} C_{\alpha, \beta}$, and $C_{14}=\frac{1}{2} \underline{C}$, we obtain

$\frac{1}{2} \frac{d}{d t}\left(\left\|\partial_{t t} v\right\|_{L^{2}(\Omega)}^{2}+\left\|\partial_{t t} \mathbf{w}\right\|_{L^{2}(\Omega)^{k}}^{2}+\left\|\partial_{t t} \mathbf{z}\right\|_{L^{2}(\Omega)^{m}}^{2}\right)+\underline{\underline{C}}\left(\left\|\nabla \partial_{t t} v+\nabla \partial_{t t} u_{e}\right\|_{L^{2}(\Omega)}^{2}+\left\|\nabla \partial_{t t} u_{e}\right\|_{L^{2}(\Omega)}^{2}+\left\|\partial_{t t} \mathbf{w}\right\|_{L^{2}(\Omega)^{k}}^{2}\right.$

$$
\left.+\left\|\partial_{t t} \mathbf{z}\right\|_{L^{2}(\Omega)^{m}}^{2}\right) \leq C_{15}\left(\left\|\partial_{t} v\right\|_{L^{2}(\Omega)}^{2}+\left\|\partial_{t} \mathbf{w}\right\|_{L^{2}(\Omega)^{k}}^{2}+\left\|\partial_{t} \mathbf{z}\right\|_{L^{2}(\Omega)^{m}}^{2}+\left\|\partial_{t t} I_{i}^{s}\right\|_{L^{2}(\Omega)}^{2}+\left\|\partial_{t t} I_{e}^{s}\right\|_{L^{2}(\Omega)}^{2}\right), 7,4
$$

where $\underline{\underline{C}}=\min \left\{\frac{1}{2} C_{\alpha, \beta}, \frac{1}{2} \underline{\underline{C}}, \frac{1}{4} \underline{\sigma_{i}}, \frac{1}{2} \underline{\sigma_{e}}\right\}$. Using hypothesis (7.34) and inequality (7.19), for $t \in[0, T]$, we integrate over the time interval $(0, t)$, we get

$$
\begin{aligned}
& \left\|\partial_{t t} v\right\|_{L^{2}(\Omega)}^{2}+\left\|\partial_{t t} \mathbf{w}\right\|_{L^{2}(\Omega)^{k}}^{2}+\left\|\partial_{t t} \mathbf{z}\right\|_{L^{2}(\Omega)^{m}}^{2}+\underline{\underline{C}} \int_{0}^{t}\left(\left\|\nabla \partial_{t t}\left(v+u_{e}\right)\right\|_{L^{2}(\Omega)}^{2}+\left\|\nabla \partial_{t t} u_{e}\right\|_{L^{2}(\Omega)}^{2}\right. \\
& \left.+\left\|\partial_{t t} \mathbf{w}\right\|_{L^{2}(\Omega)^{k}}^{2}+\left\|\partial_{t t} \mathbf{z}\right\|_{L^{2}(\Omega)^{m}}^{2}\right) d \tau \leq C\left(\left\|\partial_{t t} I_{i, e}^{S}\right\|_{L^{2}\left(0, T ; L^{2}(\Omega)\right)}^{2}+C_{15} C_{0} T+\left\|\partial_{t t} v(t=0)\right\|_{L^{2}(\Omega)}^{2}\right. \\
& \left.+\left\|\partial_{t t} \mathbf{w}(t=0)\right\|_{L^{2}(\Omega)^{k}}^{2}+\left\|\partial_{t t} \mathbf{z}(t=0)\right\|_{L^{2}(\Omega)^{m}}^{2}\right) .
\end{aligned}
$$

We obtain

$$
v, u_{e} \in H^{2}\left(0, T ; H^{1}(\Omega)\right), \quad \mathbf{w} \in H^{2}\left(0, T ; L^{2}(\Omega)\right)^{k}, \quad \text { and } \quad \mathbf{z} \in H^{2}\left(0, T ; L^{2}(\Omega)\right)^{m} .
$$

On the other hand, from the system $(2.10)$, we have

$$
\begin{aligned}
\operatorname{div}\left(\boldsymbol{\sigma}_{e} \nabla \partial_{t} u_{e}\right)= & -\partial_{t t} v-\partial_{t} I_{i o n}-\partial_{t} I_{e}^{s} \in L^{2}\left(0, T ; L^{2}(\Omega)\right), \\
& \Rightarrow u_{e} \in H^{1}\left(0, T ; H^{2}(\Omega)\right) .
\end{aligned}
$$

and

$$
\begin{gathered}
\operatorname{div}\left(\boldsymbol{\sigma}_{i} \nabla \partial_{t} v\right)=\partial_{t t} v+\partial_{t} I_{i o n}-\operatorname{div}\left(\boldsymbol{\sigma}_{i} \nabla \partial_{t} u_{e}\right)-\partial_{t} I_{i}^{s} \in L^{2}\left(0, T ; L^{2}(\Omega)\right), \\
\Rightarrow v \in H^{1}\left(0, T ; H^{2}(\Omega)\right) .
\end{gathered}
$$

Deriving (7.44) and (7.45) with respect to space, we obtain that

$$
\begin{aligned}
\partial_{x}\left[\operatorname{div}\left(\boldsymbol{\sigma}_{e} \nabla \partial_{t} u_{e}\right)\right]= & -\partial_{x} \partial_{t t} v-\partial_{x} \partial_{t} I_{i o n}-\partial_{x} \partial_{t} I_{e}^{s} \in L^{2}\left(0, T ; L^{2}(\Omega)\right) \\
& \Rightarrow u_{e} \in H^{1}\left(0, T ; H^{3}(\Omega)\right) .
\end{aligned}
$$

and

$$
\begin{gathered}
\partial_{x}\left[\operatorname{div}\left(\boldsymbol{\sigma}_{i} \nabla \partial_{t} v\right)\right]=\partial_{x} \partial_{t t} v+\partial_{x} \partial_{t} I_{i o n}-\partial_{x}\left[\operatorname{div}\left(\boldsymbol{\sigma}_{i} \nabla \partial_{t} u_{e}\right)\right]-\partial_{x} \partial_{t} I_{i}^{s} \in L^{2}\left(0, T ; L^{2}(\Omega)\right) \\
\Rightarrow v \in H^{1}\left(0, T ; H^{3}(\Omega)\right) .
\end{gathered}
$$


Let us now prove the regularities (2.22). Getting second derivatives of equations (2.5) and (2.7) over the space variable $x$, multiplying both equations by $\partial_{x x} w_{j}$ and $\partial_{x x} z_{i}$ respectively and using that fact that $\bar{\varrho}$ is bounded in $H^{3}(\Omega)^{N}$, integrating the sum over $\Omega$ and applying the Young inequality of the right hand side, we obtain

$$
\frac{d}{d t}\left(\left\|\partial_{x x} \mathbf{w}\right\|_{L^{2}(\Omega)^{k}}^{2}+\left\|\partial_{x x} \mathbf{z}\right\|_{L^{2}(\Omega)^{m}}^{2}\right) \leq C_{1}\left(\left\|\partial_{x x} \mathbf{w}\right\|_{L^{2}(\Omega)^{k}}^{2}+\left\|\partial_{x x} \mathbf{z}\right\|_{L^{2}(\Omega)^{m}}^{2}\right)+C_{2},
$$

where $C_{1}$ and $C_{2}$ are two non negative constants. Applying Gronwall Lemma, we obtain

$$
\left\|\partial_{x x} \mathbf{w}\right\|_{L^{2}(\Omega)^{k}}^{2}+\left\|\partial_{x x} \mathbf{z}\right\|_{L^{2}(\Omega)^{m}}^{2} \leq C\left(\left\|\partial_{x x} \mathbf{w}_{0}\right\|_{L^{2}(\Omega)^{k}},\left\|\partial_{x x} \mathbf{z}_{0}\right\|_{L^{2}(\Omega)^{m}}\right) .
$$

Thus,

$$
\mathbf{w} \in L^{\infty}\left(0, T ; H^{2}(\Omega)\right)^{k}, \quad \text { and } \quad \mathbf{z} \in L^{\infty}\left(0, T ; H^{2}(\Omega)\right)^{m} .
$$

Similarly, computing the third space derivatives of (2.5) and (2.7), multiplying both equations by $\partial_{x x x} w_{j}$ and $\partial_{x x x} z_{i}$ respectively and using that fact that $\bar{\varrho}$ is bounded in $H^{3}(\Omega)^{N}$, integrating the sum over $\Omega$ and applying the Young inequality of the right hand side, we obtain

$$
\frac{d}{d t}\left(\left\|\partial_{x x x} \mathbf{w}\right\|_{L^{2}(\Omega)^{k}}^{2}+\left\|\partial_{x x x} \mathbf{z}\right\|_{L^{2}(\Omega)^{m}}^{2}\right) \leq C_{3}\left(\left\|\partial_{x x x} \mathbf{w}\right\|_{L^{2}(\Omega)^{k}}^{2}+\left\|\partial_{x x x} \mathbf{z}\right\|_{L^{2}(\Omega)^{m}}^{2}\right)+C_{4}
$$

since $v \in L^{\infty}\left(0, T ; H^{3}(\Omega)\right)$, where $C_{3}$ and $C_{4}$ are two non negative constants. Using $\mathbf{w}_{0} \in H^{3}(\Omega)^{k}, \mathbf{z}_{0} \in H^{3}(\Omega)^{m}$ and applying Gronwall Lemma, we have

$$
\left\|\partial_{x x x} \mathbf{w}\right\|_{L^{2}(\Omega)^{k}}^{2}+\left\|\partial_{x x x} \mathbf{z}\right\|_{L^{2}(\Omega)^{m}}^{2} \leq C\left(\left\|\partial_{x x x} \mathbf{w}_{0}\right\|_{L^{2}(\Omega)^{k}},\left\|\partial_{x x x} \mathbf{z}_{0}\right\|_{L^{2}(\Omega)^{m}}\right)
$$

Then

$$
\mathbf{w} \in L^{\infty}\left(0, T ; H^{3}(\Omega)\right)^{k}, \quad \text { and } \quad \mathbf{z} \in L^{\infty}\left(0, T ; H^{3}(\Omega)\right)^{m} .
$$

Moreover, from the expressions of $\partial_{t}\left(\partial_{x x x} \mathbf{w}\right)$ and $\partial_{t}\left(\partial_{x x x} \mathbf{z}\right)$, we deduce

$$
\begin{aligned}
& \mathbf{w} \in W^{1, \infty}\left(0, T ; H^{3}(\Omega)\right)^{k} \subset H^{1}\left(0, T ; H^{3}(\Omega)\right)^{k} \hookrightarrow \mathcal{C}^{0}\left([0, T] ; \mathcal{C}^{1}(\bar{\Omega})\right)^{k} \\
& \mathbf{z} \in W^{1, \infty}\left(0, T ; H^{3}(\Omega)\right)^{m} \subset H^{1}\left(0, T ; H^{3}(\Omega)\right)^{m} \hookrightarrow \mathcal{C}^{0}\left([0, T] ; \mathcal{C}^{1}(\bar{\Omega})\right)^{m}
\end{aligned}
$$

The proof of Proposition 2.4 is finished.

\section{REFERENCES}

[1] E. Abbate, M. Boulakia, Y. Coudière, J.F. Gerbeau, P. Zitoun, and N. Zemzemi. In silico assessment of the effects of various compounds in mea/hipsc-cm assays: Modeling and numerical simulations. Journal of Pharmacological and Toxicological Methods, 89:59-72, 2018.

[2] Y. Abidi, M. Bellassoued, M. Mahjoub, and N. Zemzemi. On the identification of multiple space dependent ionic parameters in cardiac electrophysiology modelling. Inverse Problems, 34(3):035005, 2018.

[3] R.A. Adams. Sobolev spaces. Academic Press, New York, 1975.

[4] B. E. Ainseba, M. Bendahmane, and H. Yuan. Stability of conductivities in an inverse problem in the reaction-diffusion system in electrocardiology. American Institute of Mathematical Sciences, 10(2):369-385, 2015.

[5] G. W. Beeler and H. Reuter. Reconstruction of the action potential of ventricular myocardial fibres. The Journal of Physiology, 268(1):177-210, 1977.

[6] M. Bellassoued. Global logarithmic stability in inverse hyperbolic problem by arbitrary boundary observation. Inverse Problems, 20(4):1033-1052, 2004.

[7] M. Bellassoued and M. Yamamoto. Logarithmic stability in determination of a coefficient in an acoustic equation by arbitrary boundary observation. J. Math. Pures Appl. (9), 85(2):193-224, 2006.

[8] M. Bellassoued and M. Yamamoto. Carleman estimate and inverse source problem for biot's equations describing wave propagation in porous media. Inverse Problems, 29(115002):20pp, 2013. 
[9] A. Benabdallah, M. Cristofol, P. Gaitan, and M. Yamamoto. Inverse problem for a parabolic system with two components by measurements of one component. Applicable Analysis, 88(5):683-709, 2009.

[10] M. Boulakia and E. Schenone. Stability estimates for some parameters of a reaction-diffusion equation coupled with an ode. Applicable Analysis, 96(7):1138-1145, 2017.

[11] Y. Bourgault, Y. Coudière, and C. Pierre. Existence and uniqueness of the solution for the bidomain model used in cardiac electrophysiology. Nonlinear Analysis: Real World Applications, 10(1):458-482, 2009.

[12] A. Buen-Orovio, E. Cherry, and F. H. Fenton. Minimal model for human ventricular action potentials in tissue. Journal of Theoretical Biology, 253(3):544-560, 2008.

[13] A. L. Bukhgeim and M. V. Klibanov. Global uniqueness of class of multidimensional inverse problems, volume 24. Soviet Mathematics. Doklady, 1981.

[14] D. Chae, O. Yu. Imanuvilov, and S. M. Kim. Exact controllability for semilinear parabolic equations with neumann boundary conditions. Journal of Dynamical and Control Systems, 2(4):449-483, 1996.

[15] F. W. Chaves-Silva and M. Bendahmane. Uniform null controllability for a degenerating reaction-diffusion system approximating a simplified cardiac model. SIAM Journal on Control and Optimization, 53(6):3483-3502, 2015.

[16] Marc Courtemanche, Rafael J Ramirez, and Stanley Nattel. Ionic mechanisms underlying human atrial action potential properties: insights from a mathematical model. American Journal of Physiology-Heart and Circulatory Physiology, 275(1):H301H321, 1998.

[17] M. Cristofol, P. Gaitan, and H. Ramoul. Inverse problems for a $2 \times 2$ reaction-diffusion system using a carleman estimate with one observation. Inverse Problems, 22(5):1561, 2006.

[18] O. Yu. Emanuilov. Controllability of parabolic equations. Sbornik: Mathematics, 186(6):879-900, 1995.

[19] L. P. Endresen, K. Hall, J. S. Hoye, and J. Myrheim. A theory for the membrane potential of living cells. European Biophysics Journal, 29(2):90-103, May 2000.

[20] J. Fan and K.C Chen. Stability estimate for a strongly coupled parabolic system. Tamkang Journal of Mathematics, 43(1), 2012.

[21] Enrique Fernández-Cara, J Limaco, and SB de Menezes. Controlling linear and semilinear systems formed by one elliptic and two parabolic pdes with one scalar control. ESAIM: Control, Optimisation and Calculus of Variations, 22(4):1017-1039, 2016.

[22] D. D. Francesco and D. Noble. A model of cardiac electrical activity incorporating ionic pumps and concentration changes. Philosophical Transactions of the Royal Society of London B: Biological Sciences, 307(1133):353-398, 1985.

[23] A. V. Fursikov and O. Yu. Imanuvilov. Controllability of evolution equations, volume 34. Seoul National University, 1996.

[24] C. S. Henriquez. Simulating the electrical behavior of cardiac tissue using the bidomain model. Critical reviews in biomedical engineering, 21(1):1-77, 1993.

[25] A. L. Hodgkin and A. F. Huxley. A quantitative description of membrane current and its application to conduction and excitation in nerve. The Journal of Physiology, 117(4):500-544, 1952.

[26] O. Yu. Imanuvilov, V. Isakov, and M. Yamamoto. An inverse problem for the dynamical lamé system with two sets of boundary data. Communications on pure and applied mathematics, 56(9):1366-1382, 2003.

[27] O. Yu. Imanuvilov and M. Yamamoto. Lipschitz stability in inverse parabolic problems by the Carleman estimate. Inverse Problems, 14(5):1229-1245, 1998.

[28] O. Yu. Imanuvilov and M. Yamamoto. Determination of a coefficient in an acoustic equation with a single measurement. Inverse Problems, 19(1):157-171, 2003.

[29] V. Isakov. Inverse problems for partial differential equations, volume 127 of Applied Mathematical Sciences. Springer-Verlag, New York, 1998.

[30] T. Kato. Perturbation Theory for Linear Operators, volume 132. Springer-Verlag Berlin Heidelberg, 1995.

[31] J. P. Keener and J. Sneyd. Mathematical Physiology, I: Cellular Physiology. Graduate Studies in Mathematics. Springer-Verlag New York, 2009.

[32] M. V. Klibanov and A. A. Timonov. Carleman estimates for coefficient inverse problems and numerical applications. Utrecht: VSP, 46, 2004.

[33] J. Lassoued, M. Mahjoub, and N. Zemzemi. Stability results for the parameter identification inverse problem in cardiac electrophysiology. Inverse Problems, 32(11), 2016.

[34] P. Li and Y. Rudy. A model of canine purkinje cell electrophysiology and ca2+ cycling. Circulation Research, 2011.

[35] J. L. Lions and E. Magnenes. Non-homogeneous Boundary Value Problems and Applications, volume 1. Springer Science \& Business Media, 2012.

[36] C. H. Luo and Y. Rudy. A model of the ventricular cardiac action potential: Depolarization, repolarization, and their interaction. Circulation Research, 68(6):1501-1526, 1991.

[37] C. H. Luo and Y. Rudy. A dynamic model of the cardiac ventricular action potential. i. simulations of ionic currents and concentration changes. Circulation Research, 74(6):1071-1096, 1994.

[38] C.C. McIntyre and W. M. Grill. Sensitivity analysis of a model of mammalian neural membrane. Biological Cybernetics, 79(1):29-37, Aug 1998.

[39] D. Noble. Cardiac action and pacemaker potentials based on the hodgkin-huxley equations. Nature Publishing Group, 188(4749):495-497, 1960 
[40] T. O'Hara, L. Virág, A. Varró, and Y. Rudy. Simulation of the undiseased human cardiac ventricular action potential: Model formulation and experimental validation. PLOS Computational Biology, 7(5):1-29, 052011.

[41] M. Paci, J. Hyttinen, and S. Severi. Computational modelling of lqt1 in human induced pluripotent stem cell derived cardiomyocytes. Computing in Cardiology Conference, pages 1239-1242, 2013.

[42] M. Paci, S. Severi, and J. Hyttinen. Computational modeling supports induced pluripotent stem cell-derived cardiomyocytes reliability as a model for human lqt3. Computing in Cardiology Conference, pages 69-72, 2014.

[43] P. C. Franzone L. F. Pavarino and S. Scacchi. Mathematical Cardiac Electrophysiology. Springer International Publishing, 2014.

[44] K. Sakthivel, N. Baranibalan, J.-H. Kim, and K. Balachandran. Stability of diffusion coefficients in an inverse problem for the lotka-volterra competition system. Acta Applicandae Mathematicae, 111(2):129-147, 2010.

[45] R. M. Shaw and Y. Rudy. Electrophysiologic effects of accute myocardial ischemia: Theoretical study of altered cell exitability and action potential duration. Cardio vascular Research, 35:256-272, 1997.

[46] K. H. W. J. ten Tusscher, D. Noble, P. J. Noble, and A. V. Panfilov. A model for human ventricular tissue. American Journal of Physiology-Heart and Circulatory Physiology, 286(4):H1573-H1589, 2004. PMID: 14656705.

[47] L. Tung. A bidomain model for describing ischemic myocardial d-c potentials. PhD thesis, Massachusetts Institute of Technology, 1978

[48] M. Veneroni. Reaction-diffusion systems for the microscopic cellular model of the cardiac electric field. Mathematical methods in the applied sciences, 29(14):1631-1661, 2006.

[49] M. Veneroni. Reaction-diffusion systems for the macroscopic bidomain model of the cardiac electric field. Nonlinear Analysis: Real World Applications, 10:849-868, 2009.

[50] B. Wu and J. Liu. Determination of an unknown source for a thermoelastic system with a memory effect. Inverse Problems, 28(095012):(17pp), 2012

[51] B. Wu, L. Yan, Y. Gao, and Q. Chen. Carleman estimate for a linearized bidomain model in electrocardiology and its applications. Nonlinear Differential Equations and Applications NoDEA, 25(1):4, 2018.

[52] B. Wu and J. Yu. Hölder stability of an inverse problem for a strongly coupled reaction-diffusion system. IMA Journal of Applied Mathematics, 82(2):424-444, 2017.

[53] G. Yuan and M. Yamamoto. Lipschitz stability in the determination of the principal part of a parabolic equation. ESAIM: Control, Optimisation and Calculus of Variations, 15(3):525-554, 2009.

[54] N. Zemzemi, M. O. Bernabeu, J. Saiz, J. Cooper, P. Pathmanathan, G. R. Mirams, J. Pitt-Francis, and B. Rodriguez. Computational assessment of drug-induced effects on the electrocardiogram: from ion channel to body surface potentials. British Journal of Pharmacology, 168(3):718-733, 2013. 